\title{
A dual-layer supervised Mahalanobis kernel for the classification of hyperspectral images
}

\author{
Li Li, Chao Sun*, Lianlei Lin, Junbao Li, Shouda Jiang \\ Department of Automatic Test and Control, Harbin Institute of Technology, Harbin, China
}

\begin{abstract}
To address the drawback of traditional Mahalanobis distance metric learning (DML) methods that learn the matrix without considering the weights of each class, in this paper, a novel dual-layer supervised Mahalanobis kernel is proposed for the classification of hyperspectral images. By modifying the traditional unsupervised Mahalanobis kernel, a supervised Mahalanobis matrix that can include more relativity information of different types of real materials in hyperspectral images is learned to obtain a new kernel. The proposed Mahalanobis matrix is obtained in two steps. In step one, we learn the first traditional Mahalanobis matrix with all samples to map the raw data. In step two, based on the data mapped by the first matrix, we pick several hard-to-identify classes from all the classes and learn the second Mahalanobis matrix using only these data. Finally, by combining these two matrices, we construct a new form of the Mahalanobis kernel. Simulation experiments are conducted on three real hyperspectral data sets. We use SVM as the kernel-based classifier to classify the dimensionally reduced data and compare with several methods from various aspects. The results show that the proposed methods perform better than other unsupervised or single-layer DML methods in classifying the hard-to-identify classes, especially under an extreme condition.
\end{abstract}

Keywords: hyperspectral image classification, Mahalanobis kernel, supervised learning, SVM.

\section{Introduction}

Hyperspectral images provide a precise representation of the earth's surface with high geometrical precision and a high level of thematic detail[1]. In practice, hyperspectral image classification is a problem wherein we have to process high-dimensional data with insufficient a priori information. This problem can also result

5 in the curse of dimensionality, the so-called Hughes phenomenon, and can produce overfitting estimations 2]. Therefore, in general, the major tasks of hyperspectral image (HSI) classification are twofold: extracting essential features from enormous bands to reduce the dimension and designing suitable classifiers to obtain significant classification accuracies.

In the past decade, kernel-based classifiers have attracted increased attention because of their excellent performance when addressing high-dimensional data. Among them, the support vector machine (SVM) classifier

\footnotetext{
* Chao Sun

Email address: hitsc@163.com (Shouda Jiang)
}

Preprint submitted to Neurocomputing

May 31, 2016

(C) 2016. This manuscript version is made available under the Elsevier user license

http://www.elsevier.com/open-access/userlicense/1.0/ 
is the most representative such classifier [3, 4, 5. Many studies in the field of remote sensing have addressed the fact that SVM-based classifiers provide superior performance in hyperspectral data classification [6, 7, 8, 9, 10] compared with other popular classifiers such as the k-nearest neighbors (KNN) classifier and artificial neural networks (ANNs).

To improve the generalization capability of kernel-based learning machines (such as SVMs and SRCs [11]), different forms of optimized-kernel methods have been proposed. One form consists of constructing multi-kernel learning (MKL) methods [12, 13, 14, 15, 16, 17, 18, 19, 20, 21. However, the solution for identifying the weight of each kernel is always obtained by solving an optimization problem, such as a semi-definite program (SDP) [12] or semi-infinite linear program (SILP) problem [22, which are excessively computationally complex. Another shortcoming is that they do not account for the statistical regularities of the specific classification task and are consequently susceptible to noise or irrelevant spectral features.

The other form is to construct a kernel that can increase the spatial resolution near the separating boundary surface; a representative family of such methods is data-dependent kernels 223 24, and another family of methods consists of adopting distance metric learning (DML) methods. In recent years, there has been a growing body of work in the field of metric learning on the development of adaptive similarity measures that learn the relevances of features with respect to a given classification task [25, 26, 27]. Substituting an unweighted measure with a learned similarity measure offers a straightforward means of improving the classification accuracy with similarity-based classifiers. In addition, the DML algorithms have already been extended with kernel tricks 28, 29, 30, 31, 32, 33, 34. Among these metric learning methods, the Mahalanobis metric learning method has been found to provide the best performance 35, 36, 37, 38, 39, 31, 40, 41, 42, Its expression is given by Eq, 1 and the goal is to compute a linear projection matrix $M$. Applying $A$ to each sample pair $x_{i}, x_{j}$ induces a Mahalanobis distance parametrized by the PSD matrix $M=A A^{T}$.

$$
\begin{aligned}
d_{M}\left(x_{i}, x_{j}\right) & =\sqrt{\left(A^{T} x_{i}-A^{T} x_{j}\right)^{T}\left(A^{T} x_{i}-A^{T} x_{j}\right)} \\
& =\sqrt{\left(x_{i}-x_{j}\right)^{T} A A^{T}\left(x_{i}-x_{j}\right)} \\
& =\sqrt{\left(x_{i}-x_{j}\right)^{T} M\left(x_{i}-x_{j}\right)}
\end{aligned}
$$

Beyond the full-rank Mahalanobis distances, increasingly more works propose techniques to learn lowrank Mahalanobis distances. A low-rank Mahalanobis metric learning method is used to project samples from the original-dimensional feature space into a lower dimensional feature space. Low-rank matrices are particularly well-suited for hyperspectral image classification problems because of the high dimensionality of hyperspectral data and the limited amounts of training data. Moreover, the reduced dimensionality reduces the computational time and memory requirements [43, 44]. In [45], Gomez and Camps-Valls proposed the Mahalanobis kernel (MK) in the formulation of the SVM and applied it to the field of hyperspectral classification. In addition, in [46, D. Bue introduced several typical Mahalanobis methods into the SVM and evaluated the resulting performance.

However, a drawback of the normal Mahalanobis kernel is that such methods simply apply the same 
weight to all the samples to learn the Mahalanobis matrix and cannot learn a matrix to increase the distance to concentrate on the partial features of several special classes.

Recently, through various experiments, we discovered some phenomena that can be observed in different hyperspectral images. Specifically, in a given hyperspectral image, when we remove one or several labels, another class's classification accuracy can greatly increase, whereas the accuracy of the remaining classes is hardly affected. This means that, for a certain hyperspectral image, there are always several classes whose clusters are quite close to some classes but sufficiently far from other classes, which makes it such that the samples in these classes can have one or several incorrect labels easily attached to them but not to the remaining samples.

Thus, in this paper, considering this property of hyperspectral images, by analyzing the drawback of the current Mahalanobis kernel, we propose a novel dual-layer supervised Mahalanobis DML method to learn a new form of the Mahalanobis matrix $M$ and to construct a new Mahalanobis kernel. In contrast to current methods, and going beyond the method proposed in [40, where the author proposed a weighted-NCA method in which a weighted matrix is generated to tune the weights of different classes but in which a quantization method is not proposed, using an indiscriminate Mahalanobis DML on the first layer, we extract more precise relevancies of features between different classes and determine the potentially hard-to-identify classes. We then re-cluster these data by learning a second Mahalanobis matrix. Considering computational efficiency, we adopt an LDA-based Mahalanobis DML algorithm to compute the transformation matrix. Numerical experiments are conducted on several real hyperspectral data sets. To evaluate the performance of our proposed methods, we compare our methods with some single- and state-of-the-art multi-kernel methods based on various aspects. The experiments show that, with the proposed methods, we can increase the partial difference between the special classes better than we can under the traditional Mahalanobis kernel while simultaneously having little negative effect on the remaining classes.

The remainder of this paper is organized as follows. In Section III, we introduce the proposed methods in detail. Numerical experimental results on three different real-world hyperspectral data sets are presented in Section III. Finally, some conclusions are drawn from the experiments, and suggestions for future work are given in Section IV.

\section{Algorithm}

\subsection{Mahalanobis kernel for SVM}

Given a labeled training data set $\left\{\left(x_{1}, y_{1}\right), \ldots,\left(x_{n}, y_{n}\right)\right\}$, where $x_{i} \in \mathbb{R}^{N}$ and $y_{i} \in\{-1,+1\}$, and a nonlinear mapping $\phi($.$) , usually to a higher (possibly infinite) dimensional Hilbert space, \phi: \mathbb{R}^{N} \rightarrow \mathcal{H}$, the SVM method solves

$$
\min _{w, \zeta_{i}, b}\left\{\frac{1}{2}\|w\|^{2}+C \sum_{i} \zeta_{i}\right\}
$$


with the constraint

$$
\begin{aligned}
y_{i}\left(\left\langle\phi\left(x_{i}, w\right)\right\rangle+b\right) & \geq 1-\zeta_{i} \quad \forall i=1, \ldots, n \\
\zeta_{i} & \geq 0
\end{aligned}
$$

where $w$ and $b$ define a linear classifier in the feature space. The nonlinear mapping function $\phi$ is applied in accordance with Cover's theorem[47, which guarantees that the transformed samples are more likely to be linearly separable in the resulting feature space. The regularization parameter $C$ controls the generalization capabilities of the classifier and must be selected by the user, and $\zeta_{i}$ are positive slack variables enabling 80 permitted errors to be addressed.

It is worth noting that all $\phi$ mappings used in the SVM learning occur in the form of inner product$\mathrm{s}$ in $\mathcal{H}$, which allows us to define a kernel function $K\left(x_{i}, x_{j}\right)=\left\langle\phi\left(x_{i}\right), \phi\left(y_{i}\right)\right\rangle$. Then, a nonlinear SVM can be constructed using only the kernel function without having to consider the mapping $\phi$ explicitl[48]. Finally, the decision function implemented by the classifier for any test vector $x_{*}$ is given by $f\left(x_{*}=\right.$ $\left.{ }_{85} \operatorname{sgn}\left(\sum_{i=1}^{n} y_{i} \alpha_{i} K\left(x_{i}, x_{j}\right)+b\right)\right)$, where $b$ can be easily computed from the $\alpha_{i}$ that are neither 0 nor $C$.

Gomez and Camps-Valls [4] introduced the Mahalanobis kernel in the formulation of the SVM, which is based on an RBF kernel $K\left(x_{i}, x_{j}\right)=\exp \left(-1 / 2 \sigma^{2}\left(\left\|x_{i}-x_{j}\right\|^{2}\right)\right)$ and is defined as

$$
K\left(x_{i}, x_{j}\right)=\exp \left(-\frac{1}{2 \sigma^{2}}\left(x_{i}-x_{j}\right)^{T} M^{-1}\left(x_{i}-x_{j}\right)\right)
$$

where $M$ is the estimated Mahalanobis distance metric matrix computed using the available training data. Note that this constitutes a nonlinear generalization of any Mahalanobis distance metric through the use of the kernel method's framework.

\subsection{Mahalanobis DML algorithm}

\subsubsection{Related work}

Suppose we have two data points $x_{1}, x_{2} \in \mathbb{R}^{n}$, and their Mahalanobis distance can be defined as :

$$
d_{A}\left(x_{1}, x_{2}\right)=\sqrt{\left(x_{1}-x_{2}\right)^{T} A\left(x_{1}-x_{2}\right)}
$$

where $A \in \mathbb{R}^{n \times n}$ is a positive semi-definite matrix which can be decomposed into $A=W W^{T}$ by performing 95 an eigenvalue decomposition(EVD). So we can solve this problem by learning the matrix $W$ and have the following equation:

$$
d_{A}\left(x_{1}, x_{2}\right)=\sqrt{\left(x_{1}-x_{2}\right)^{T} W W^{T}\left(x_{1}-x_{2}\right)}
$$

Following Xiang[49, we solve the optimization problem by adopting a binary iterative search method. From the experiments, we can see that significant time can be saved during the classification for hyperspectral image data. 
We begin with a set of samples $\mathcal{X}=\left\{x_{i}\right\}_{i=1}^{N} \subset \mathbb{R}^{n}$ and two constraint sets, namely, the must-link pairs $\mathcal{S}=\left\{\left(x_{i}, x_{j}\right) \mid x_{i}\right.$ and $x_{j}$ are in a same class $\}$ and the cannot-link pairs $\mathcal{D}=\left\{\left(x_{i}, x_{j}\right) \mid x_{i}\right.$ and $x_{j}$ are in two different class\}. With this information, we need to learn a Mahalanobis matrix A, which is applied to map data into a new feature space. In the transformation space, the distances of points in $\mathcal{S}$ should be reduced to the greatest extent possible. In contrast, the distances between points in $\mathcal{D}$ should be increased to the greatest extent possible.

Based on Eq. (6), we can achieve this goal by optimizing the matrix $W \in \mathbb{R}^{n \times n}$, with $d \leq n$. Finally, we deduce the transformation

$$
y=W^{T} x
$$

Here, we provide the definitions of $S_{b}$ (called the between-class scatter matrix) and $S_{w}$ (the within-class scatter matrix). Assuming there are $C$ classes, we have

$$
\begin{aligned}
S_{w} & =\frac{1}{N} \sum_{j=1}^{C} \sum_{i=1}^{n_{j}}\left(x_{j i}-\tilde{x_{j}}\right)\left(x_{j i}-\tilde{x_{j}}\right)^{T} \\
S_{b} & =\frac{1}{N} \sum_{j=1}^{C} n_{j}\left(\tilde{x_{j}}-\tilde{x}\right)\left(\tilde{x_{j}}-\tilde{x}\right)^{T}
\end{aligned}
$$

where $n_{j}$ denotes the sample number of class $c, N=\sum\left(n_{j}\right)$ is the number of all samples, $x_{j i}$ denotes the $i$ th sample point in class $j, \tilde{x_{j}}$ is the mean value of class $j$, and $\tilde{x}$ is the mean value of all sample points.

Following Eq. (7), we can obtain

$$
\begin{aligned}
\hat{S_{w Y}} & =\frac{1}{N} \sum_{j=1}^{C} \sum_{i=1}^{n_{j}}\left(y_{j i}-\tilde{y_{j}}\right)\left(y_{j i}-\tilde{y_{j}}\right)^{T} \\
& =\frac{1}{m} \sum_{j=1}^{C} \sum_{i=1}^{n_{j}}\left(W^{T} x_{j i}-W^{T} \tilde{x_{j}}\right)\left(W^{T} x_{j i}-W^{T} \tilde{x_{j}}\right)^{T} \\
& =W^{T}\left(\frac{1}{N} \sum_{j=1}^{C} \sum_{i=1}^{n_{j}}\left(x_{j i}-\tilde{x_{j}}\right)\left(x_{j i}-\tilde{x_{j}}\right)^{T}\right) W \\
& =W^{T} \hat{S_{w}} W
\end{aligned}
$$




$$
\begin{aligned}
\hat{S_{b Y}} & =\frac{1}{N} \sum_{j=1}^{C} n_{j}\left(\tilde{y}_{j}-\tilde{y}\right)\left(\tilde{y}_{j}-\tilde{y}\right)^{T} \\
& =\frac{1}{N} \sum_{j=1}^{C} n_{j}\left(W^{T} \tilde{x_{j}}-W^{T} \tilde{x}\right)\left(W^{T} \tilde{x_{j}}-W^{T} \tilde{x}\right)^{T} \\
& =W^{T}\left(\frac{1}{N} \sum_{j=1}^{C} n_{j}\left(\tilde{x_{j}}-\tilde{x}\right)\left(\tilde{x_{j}}-\tilde{x}\right)^{T}\right) W \\
& =W^{T} \hat{S_{b}} W
\end{aligned}
$$

Then, we use the trace of those matrices to measure the linear separability of class, where

$$
\begin{aligned}
\operatorname{tr}\left(\hat{S_{w Y}}\right) & =\operatorname{tr}\left(W^{T} \hat{S_{w}} W\right) \\
& =\frac{1}{N} \sum_{j=1}^{C} \sum_{i=1}^{n_{j}}\left(W^{T} x_{j i}-W^{T} \tilde{x_{j}}\right)^{T}\left(W^{T} x_{j i}-W^{T} \tilde{x_{j}}\right) \\
& =\frac{1}{N} \sum_{j=1}^{C} \sum_{i=1}^{n_{j}}\left(x_{j i}-\tilde{x_{j}}\right)^{T} W W^{T}\left(x_{j i}-\tilde{x_{j}}\right) \\
& =\frac{1}{N} \sum_{j=1}^{C} \sum_{i=1}^{n_{j}} d_{A}^{2}\left(x_{j i}, \tilde{x_{j}}\right)
\end{aligned}
$$

which is $1 / N$ times the sum of the squared Mahalanobis distances of each point to its cluster's center, indicating the separability of the point pairs in $\mathcal{S}$. Similarly, for $S_{b Y}$, we have

$$
\begin{aligned}
\operatorname{tr}\left(\hat{S_{b Y}}\right) & =\operatorname{tr}\left(W^{T} \hat{S_{b}} W\right) \\
& =\frac{1}{N} \sum_{j=1}^{C} n_{j}\left(W^{T} \tilde{x_{j}}-W^{T} \tilde{x}\right)^{T}\left(W^{T} \tilde{x_{j}}-W^{T} \tilde{x}\right) \\
& =\frac{1}{N} \sum_{j=1}^{C} n_{j}\left(\tilde{x_{j}}-\tilde{x}\right)^{T} W W^{T}\left(\tilde{x_{j}}-\tilde{x}\right) \\
& =\frac{1}{N} \sum_{j=1}^{C} n_{j} d_{A}^{2}\left(\tilde{x_{j}}, \tilde{x}\right)
\end{aligned}
$$

This trace is $1 / N$ times the sum of the squared Mahalanobis distances of each cluster's center to the center of all samples, which indicates the separability of point pairs in $\mathcal{D}$

Obviously, our goal is to minimize $\operatorname{tr}\left(\hat{S_{w Y}}\right)$ and maximize $\operatorname{tr}\left(\hat{S_{b Y}}\right)$, namely, to increase the distance between different class clusters and reduce the distance within a class to the greatest extents possible. Many algorithms achieve this goal by solving the following problem:

$$
W^{*}=\arg \max _{W^{T} W=I} \frac{\operatorname{tr}\left(W^{T} \hat{S_{b}} W\right)}{\operatorname{tr}\left(W^{T} \hat{S_{w}} W\right)}
$$


Here, $W \in \mathbb{R}^{n \times d}$ which submits to $W^{T} W=I$, where the $I \in \mathbb{R}^{(d \times d)}$ is an identity matrix. We mean to avoid the degenerate problem by introducing this constraint. It is notable that, when $d<n$, the matrix $A=W W^{T}$ is not an identity matrix, and $W$ will not be a square matrix either.

After obtaining the optimum $\mathrm{W}^{*}$, we can calculate the Mahalanobis matrix $A$ by

$$
A= \begin{cases}W^{*}\left(W^{*}\right)^{T} & \text { when } d<n \\ I & \text { when } d=n\end{cases}
$$

\subsubsection{Solution of the optimization problem}

The Problem Eq. (13) indicates that to maximize the ratio, the numerator should be decreased, and the denominator $\operatorname{tr}\left(W^{T} \hat{S_{w}} W\right)$ should be increased. We solve this problem by applying the following theorem:

Theorem 1. Given a matrix $W \in \mathbb{R}^{n \times d}$ and the constraint condition $W^{T} W=I$, denote the rank of the matrix $\hat{S_{w}}$ as $r$, where $r \leq n$. In the case $d>n-r, \operatorname{tr}\left(W^{T} \hat{S_{w}} W\right)>0$, and when $d \leq n-r, \operatorname{tr}\left(W^{T} \hat{S_{w}} W\right)=0$.

Here, we should analyze this problem from two aspects.

Condition $A: d>n-r$

Denote $\lambda^{*}$ as the optimal value of Eq. 13); therefore, we obtain

$$
\lambda^{*}=\max _{W^{T} W=I} \frac{\operatorname{tr}\left(W^{T} \hat{S_{b}} W\right)}{\operatorname{tr}\left(W^{T} \hat{S_{w}} W\right)}
$$

According to the work of Guo et al. [50], we obtain the following:

$$
\max _{W^{T} W=I} \operatorname{tr}\left(W^{T}\left(\hat{S_{b}}-\lambda^{*} \hat{S_{w}}\right) W\right)=0
$$

Then, we define a function of $g(\lambda)$ by

$$
g(\lambda)=\max _{W^{T} W=I} \operatorname{tr}\left(W^{T}\left(\hat{S_{b}}-\lambda^{*} \hat{S_{w}}\right) W\right)
$$

According to [51, the value of $g(\lambda)$ is equal to the sum of the $d$ largest eigenvalues of the matrix $\left(\hat{S}_{b}-\lambda \hat{S}_{b}\right)$. In addition, according to Eq. 16 , our goal is to search for a $\lambda$ satisfying $g(\lambda)=0$.

When $d<n-r$ and $\operatorname{tr}\left(W^{T} \hat{S_{w}} W\right)>0$, we can obtain the following propositions:

$$
\begin{aligned}
& g(\lambda)<0 \Rightarrow \lambda>\lambda^{*} \\
& g(\lambda)>0 \Rightarrow \lambda<\lambda^{*}
\end{aligned}
$$

These propositions indicate $\lambda^{*}$ can be calculated according to $g\left(\lambda^{*}\right)$ iteratively.

To find the optimum $\lambda^{*}$, we should set an initial value for the iteration first and subsequently set both the upper and lower bounds so that the method can perform a binary search to find $\lambda^{*}$. In practice, we apply the second theorem: 
Theorem 2. When $d>n-r$, letting the rank of $\hat{S_{w}}$ be r, we have

$$
\frac{\operatorname{tr}\left(\hat{S_{b}}\right)}{\operatorname{tr}\left(\hat{S_{w}}\right)} \leq \lambda^{*} \leq \frac{\sum_{i=1}^{d} \alpha_{i}}{\sum_{i=1}^{d} \beta_{i}}
$$

where $\beta_{1}, \ldots, \beta_{d}$ denote the first $d$ smallest eigenvalues of $\hat{S_{w}}$, while the $\alpha_{1}, \ldots, \alpha_{d}$ denote the first $d$ largest

145

The algorithm for learning the Mahalanobis matrix with constraint information is listed in Algorithm (2).

We perform an EVD of the matrix $\hat{S_{b}}+\hat{S_{w}}$ to calculate the null space. If the number of data points

Condition $B: d \leq n-r$

Clearly, maximizing the formulation is equivalent to minimizing the second term $\lambda \operatorname{tr}\left(W^{T} S_{w} W\right)$. Additionally, according to the Rayleigh quotient theory, $\min \operatorname{tr}\left(W^{T} S_{w} W\right)$ is equal to the sum of the first d smallest eigenvalues of $S_{w}$. Because $S_{w}$ is a PSD matrix, all its eigenvalues cannot be less than zero. Let the rank of $S_{w}$ be $r$, when $n-r$ is grater than $d$, namely, the reserved number of eigenvalues is greater than the number of zero-eigenvalues of $S_{w}$; therefore, we can always find at least one non-zero value and ensure that $\operatorname{tr}\left(S_{w}\right)>0$. Under this condition, we can obtain the maximum of $\operatorname{tr}\left(W^{T}\left(S_{b}-\lambda S_{w}\right) W\right)$ by tuning the $\lambda$ to obtain the optimum of $W^{*}$ by continuously performing eigenvalue decomposition. However, when $d<n-r$, all $d$ eigenvalues might be zero, and the trace of $S_{w}$ would be zero too, which means that we cannot find the maximum of $\operatorname{tr}\left(W^{T}\left(S_{b}-\lambda S_{w}\right) W\right)$ nor the optimum $W^{*}$ by tuning $\lambda$. Under this condition, we calculate the trace of $W^{T} S_{b} W$ directly by solving the following problem:

$$
V^{*}=\arg \max _{V^{T} V=I}\left(V^{T}\left(Z^{T} \hat{S}_{b} Z\right) V\right)
$$

where $V, Z \in \mathbb{R}^{n \times(n-r)}$, and $Z$ consists of the corresponding eigenvectors of the $n-r$ zero eigenvalues of $\hat{S_{w}}$ in column. By obtaining the $V^{*}$, we can calculate the $W^{*}$ by $W^{*}=Z V^{*}$.

What we want to emphasize is that, although this type of method can reduce the dimensionality of data in practice, based on the nature of hyperspectral data, to reserve as much information as possible, in this section of the experiment, we reduce the dimensionality by a quite limited extent during a single transformation; therefore, the condition $d<n-r$ never occurs in this paper.

The algorithm is listed in the table of Algorithm1, where we can see that EVD must be performed on the matrix $\hat{S_{b}}-\lambda \hat{S_{w}}$ continually. However, when $n$ is quite large, the task becomes too difficult for a PC to perform. Thus, reducing the data dimension is desired in this case. Then, we obtain the following theorem.

Theorem 3. Problem 13 can be solved in the orthogonal complement space of the null space of $\hat{S_{w}}+\hat{S_{b}}$ with no loss of information 49 .

(which is $N$ ) is no greater than the dimensionality $n$ and the rank of the matrix $\hat{S_{b}}+\hat{S_{w}}$ should also be no greater than $N$ either. Under this condition, we can adopt the following theorem instead of performing eigenvalue decomposition.

Theorem 4. Given two matrixes $M \in \mathbb{R}^{n \times N}$ and $N \in \mathbb{R}^{N \times n}$, the rank of $M N$ and that of $N M$ will be the same, which means that they have the same $r$ non-zero eigenvalues. Let $u$ and $v$ be the eigenvector of 
the eigenvalue $\alpha$ of $M N$ and $N M$; then, we can have $u=N v[52$.

Then, we use a matrix $X=\left[x_{1}, x_{2}, \ldots, x_{N}\right] \in \mathbb{R}^{n \times N}$ to denote the data matrix constructed by the $\mathrm{N}$ samples. For the constraints in $\mathcal{S}$, we can form a symmetrical matrix $S \in \mathbb{R}^{N \times N}$ by

$$
\begin{cases}S(i, j)=S(j, i)=1 ; & \left(x_{i}, x_{j}\right) \in \mathcal{S} \\ S(i, j)=S(j, i)=0 ; & \left(x_{i}, x_{j}\right) \notin \mathcal{S}\end{cases}
$$

Similar to the must-link point pairs $\mathcal{S}$, we also define a symmetrical matrix $D \in \mathbb{R}^{N \times N}$ that reflects the situation in the cannot-link constraint $\mathcal{D}$ :

$$
\begin{cases}D(i, j)=D(j, i)=1 ; & \left(x_{i}, x_{j}\right) \in \mathcal{D} \\ D(i, j)=D(j, i)=0 ; & \left(x_{i}, x_{j}\right) \notin \mathcal{D}\end{cases}
$$

Let $L_{b}=\operatorname{diag}(\operatorname{sum}(D))-D$ and $L_{w}=\operatorname{diag}(\operatorname{sum}(S))-S$, where $\operatorname{sum}()$ is the sum of a matrix by row. We can obtain $\hat{S_{w}}$ and $\hat{S_{b}}$ by

$$
\begin{aligned}
\hat{S_{w}} & =\frac{1}{2} X L_{w} X^{T} \\
\hat{S_{b}} & =\frac{1}{2} X L_{b} X^{T}
\end{aligned}
$$

Thus, we have

$$
\hat{S_{w}}+\hat{S_{b}}=X\left(\frac{1}{2} L_{w}+\frac{1}{2} L_{b}\right) X^{T}
$$

Note that a matrix's rank equals its number of non-zero eigenvalues [49]. Let $L=X^{T} X\left(\frac{1}{2} L_{w}+\frac{1}{2} L_{b}\right) \in$ $\mathbb{R}^{N \times N}$, and let $r$ be the rank of $L(r \leq N)$. When $n>N$, by performing an EVD, we can obtain $r$ non-zero eigenvalues and corresponding eigenvectors. Now, denote each corresponding eigenvector by $v_{i}, i=1, \ldots, r$. Based on this theorem, we can obtain the eigenvectors of $\hat{S}_{b}+\hat{S_{w}}$, namely, $u_{i}$, by performing

$$
u_{i}=X\left(\frac{1}{2} L_{w}+\frac{1}{2} L_{b}\right) v_{i}, i=1,2, \ldots, r
$$

It is notable that the length of these eigenvectors has not been normalized; therefore, we should normalize each $u_{i}$ by performing $u_{i} \leftarrow \frac{u_{i}}{\left\|u i_{i}\right\|}$ so that all these vectors are of unit length.

Now, let $W^{1}=\left[u_{1}, \ldots, u_{r}\right] \in \mathbb{R}^{n \times r}$, where the column vectors of the matrix are the $r$ normalized eigenvec190 tors of $\hat{S_{b}}+\hat{S_{w}}$. Because these $r$ vectors $\left\{u_{1}, u_{2}, \ldots, u_{r}\right\}$ are orthogonal to each other and have unit length, they can constitute a set of bases of the orthogonal complement space of the null-zero space of $\hat{S_{b}}+\hat{S_{w}}$ [49]. In addition, by applying the matrix $W^{1}$, all our data points can be projected to the space by performing 7 Using the low-rank Mahalanobis matrix, we can reduce the dimensionality of the source data. 


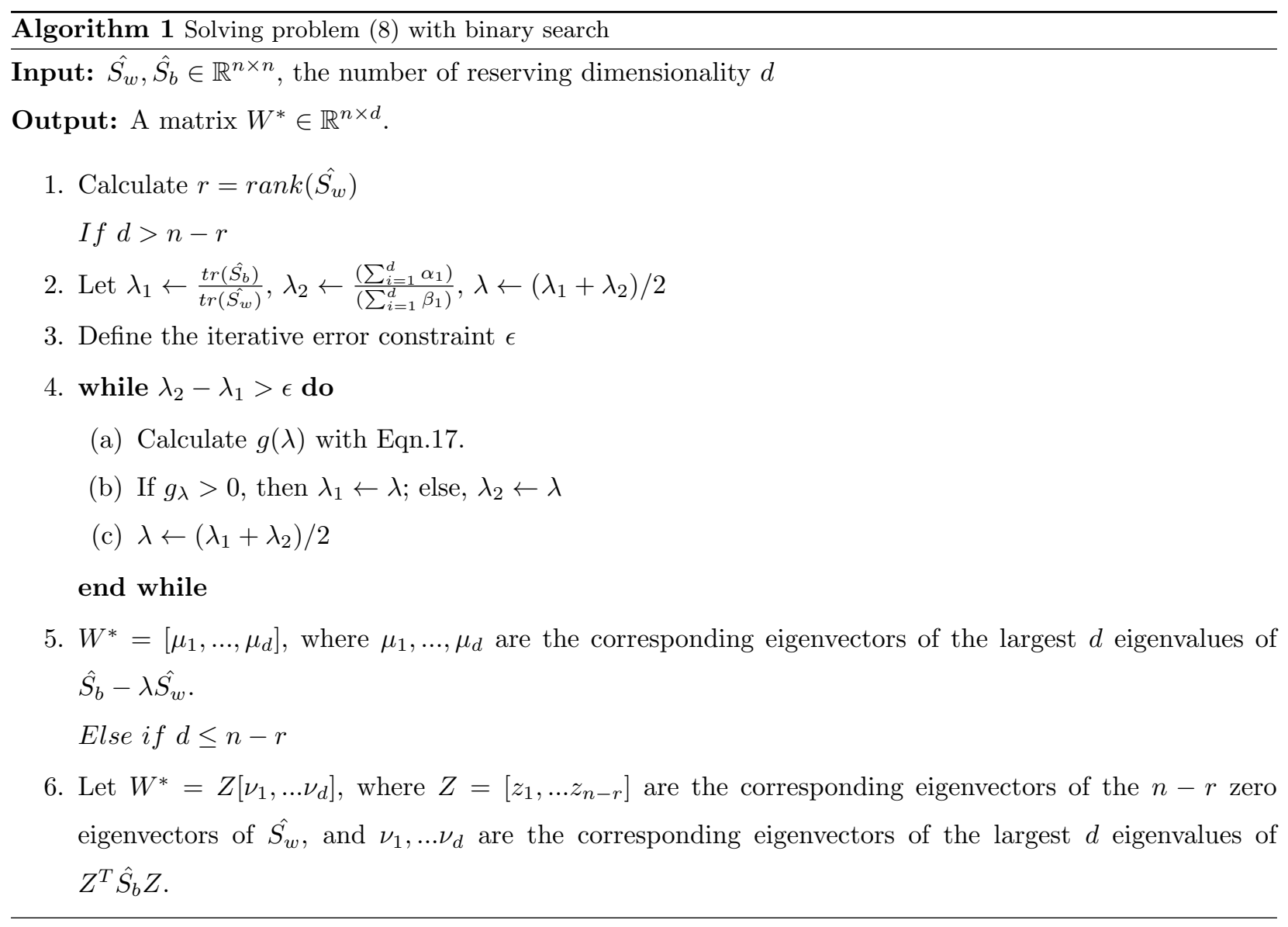

\subsection{A dual-layer supervised Mahalanobis DML strategy}

In [46], Bue introduced several classical Mahalanobis DML methods into the Mahalanobis kernel; however, these types of indiscriminate optimization methods cannot highlight the specific relationship between the hard-to-identify pairings. For hyperspectral images, if the pairing of the two classes whose distance-betweenclasses is already sufficiently large to distinguish the samples, the improvement obtained by increasing those distance-between-classes is quite limited. Meanwhile, because these unnecessary pairings are considered, the pairings that should be given greater attention are ignored relatively.

Thus, in this paper, we propose a dual-layer Mahalanobis DML method, whose formulation can be written as in Eq. 267.

$$
\begin{aligned}
d_{A}\left(x_{1}, x_{2}\right) & =\sqrt{\left(x_{1}-x_{2}\right)^{T} A\left(x_{1}-x_{2}\right)} \\
& =\sqrt{\left(x_{1}-x_{2}\right)^{T} W^{*}\left(W^{*}\right)^{T}\left(x_{1}-x_{2}\right)} \\
& =\sqrt{\left(x_{1}-x_{2}\right)^{T}(W V)(W V)^{T}\left(x_{1}-x_{2}\right)}
\end{aligned}
$$

where the matrix $W \in \mathbb{R}^{n \times(n-1)}$ is a matrix learned with all classes indiscriminately, through which we can learn the first Mahalanobis matrix and perform the first transformation. Based on this distance metric, we 
Algorithm 2 Algorithm for learning a Mahalanobis distance metric

Input: $\hat{S_{w}}, \hat{S}_{b} \in \mathbb{R}^{n \times n}$, the number of reserving dimensionality $d$

Output: The Mahalanobis matrix $A$ and transformation matrix $\hat{W}^{*}=W_{1} W^{*}$.

1. Initialization:

(a) Compute the matrix $W_{1}^{T}$, restricted by $W_{1}^{T} W_{1}=I$. Here, $W_{1}^{T}$ consists of the corresponding eigenvectors of all the non-zero eigenvalues of $\hat{S}_{b}+\hat{S_{w}}$.

(b) Calculate the new form matrices $\tilde{S_{w}}=W_{1}^{T} \hat{S_{w}} W_{1}$ and $\tilde{S_{b}}=W_{1}^{T} \hat{S_{b}} W_{1}$.

2. Learn the optimal matrix $W^{*}$ with Algorithm 1 with $\tilde{S_{w}}$ and $\tilde{S_{b}}$.

3. Output the Mahalanobis matrix: $A=W_{1} W^{*}\left(W^{*}\right)^{T} W_{1}^{T}$

observe the distance-within-class of every class and the distance-between-classes of all other classes, selectively choose several of the most "suspicious" pairings, and train a new matrix $V \in \mathbb{R}^{(n-1) \times(n-2)}$ specifically using these data for the second iteration. Through this, we can increase the distance-between-classes of the potential hard-to-identify pairings while producing minimal effects on the other data. The flow of one type of automatic selection strategy is depicted in Algorithm (3).

\section{EXPERIMENTAL RESULTS AND ANALYSIS}

\subsection{Data description}

In this set of experiments, we evaluate the classification accuracy of the proposed approach using the Indian Pines data set (AVIRIS), Salinas Valley data set (AVIRIS) and Pavia University data set (ROSIS) with various spectral and spatial resolutions reflecting the different environments found in remote sensing.

(1) Indian Pines data: These data, which have a spectral resolution of 224 bands covering the $0.4-2.5 \mu \mathrm{m}$ range and a spatial resolution of $20 \mathrm{~m}$ per pixel, were captured over the agricultural region of Northwestern Indiana in June 1992. After removing the noisy and water vapor absorption bands, 200 bands are reserved for the experiments. Although the image consists of 16 classes of interest, the numbers of pixels of 4 such classes are less than 100. In this paper, we only classify the remaining 12 classes of materials (see Fig 1), and the classification maps obtained under different methods are shown in Fig.5.

(2) Pavia University data: This data set was acquired by the Reflective Optics System Imaging Spectrometer (ROSIS) over the urban area of the University of Pavia, Northern Italy. The whole data set consists of 115 spectral bands and $610 \times 610$ pixels, with a spatial resolution of $1.3 \mathrm{~m}$ per pixel. Several undesirable bands influenced by atmospheric absorption are discarded, leaving 103 bands in the 0.43-0.86 $\mu m$ region. From the whole set, we extract a patch with a size of $610 \times 340$, which consists of 9 classes of land cover classes. The quantities of each class used in the experiments are depicted in Fig, 1, and the classification maps obtained for different methods are shown in Fig 6 . 


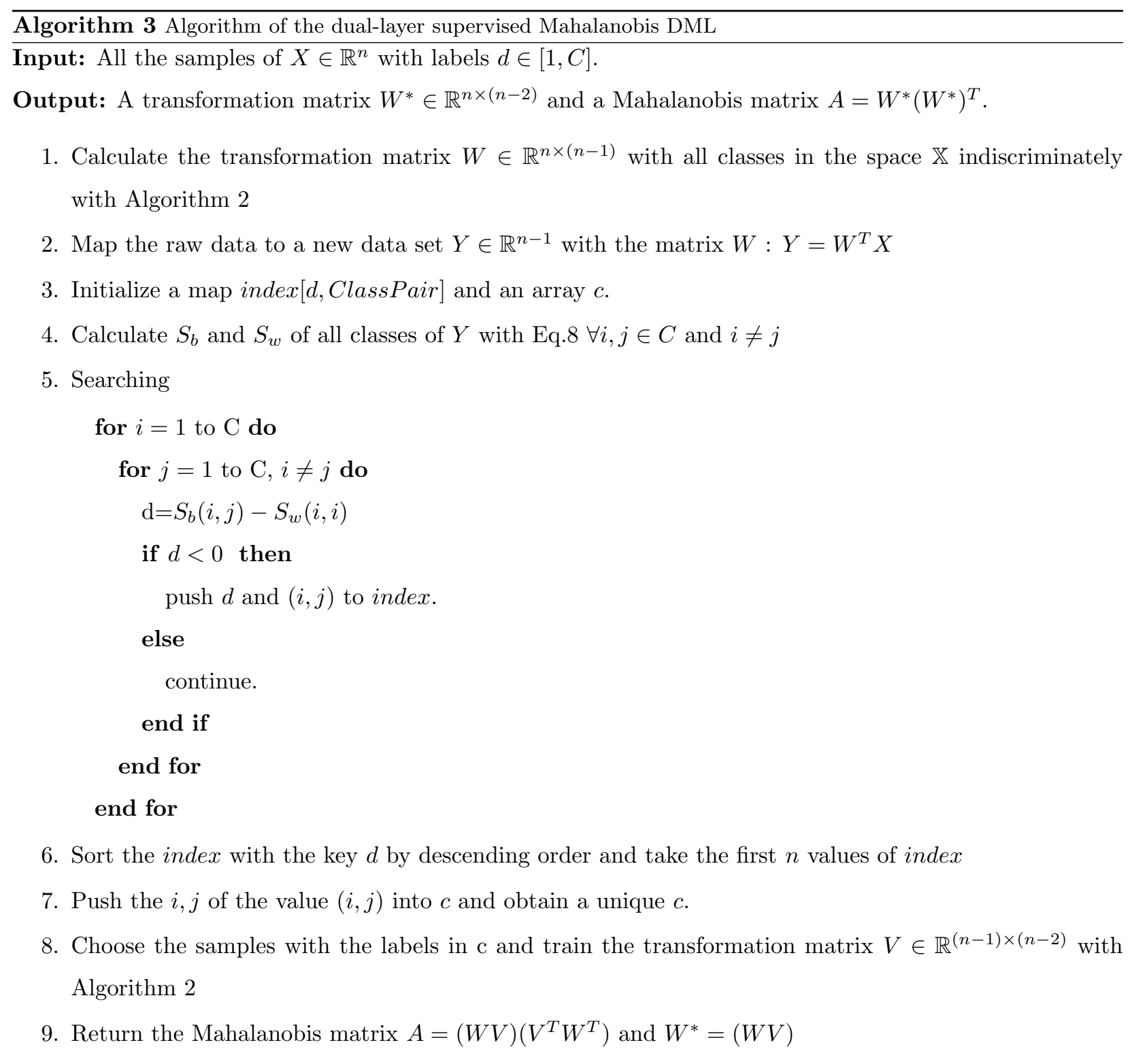


(3) Salinas Valley data: This scene was collected by the AVIRIS sensor over Salinas Valley CA, USA. It has a spatial resolution of $3.7 \mathrm{~m}$ per pixel and 224 spectral bands, with a wavelength range of 0.4-2.5 $\mu \mathrm{m}$. 16 land cover classes. The classification maps of different methods are shown in Fig.7.

\begin{tabular}{|c|c|c|c|c|c|c|}
\hline \multirow[b]{2}{*}{ CLASS } & \multicolumn{2}{|c|}{ AVIRIS Indiana Pine } & \multicolumn{2}{|c|}{ ROSIS Pavia University } & \multicolumn{2}{|l|}{ AVIRIS SALINAS } \\
\hline & Categories & Samples & Categories & Samples & Categories & Samples \\
\hline $\mathrm{C} 1$ & Corn-notill & 1434 & Asphalt & 6852 & Broccoli-green-weeds_2 & 2009 \\
\hline $\mathrm{C} 2$ & Corn-mintill & 834 & Meadows & 18686 & Broccoli-green-weeds_2 & 3726 \\
\hline $\mathrm{C} 3$ & Corn & 234 & Gravel & 2207 & Fallow & 1976 \\
\hline $\mathrm{C} 4$ & Grass-pasture & 497 & Trees & 3436 & Fallow-rough-plow & 1394 \\
\hline $\mathrm{C} 5$ & Grass-trees & 747 & Painted metal sheets & 1378 & Fallow-Smooth & 2678 \\
\hline C6 & Hay-windrowed & 489 & Bare Soil & 5104 & Stubble & 3959 \\
\hline C7 & Soybean-notill & 968 & Bitumen & 1356 & Celery & 3579 \\
\hline $\mathrm{C} 8$ & Soybean-mintill & 2468 & Self-blocking bricks & 3878 & Grapes-untrained & 11271 \\
\hline $\mathrm{C} 9$ & Soybean-clean & 614 & Shadows & 1026 & Soil-vinyary-develop & 6203 \\
\hline $\mathrm{C} 10$ & Wheat & 212 & & & Corn-sensced-green-weeds & 3278 \\
\hline $\mathrm{C} 11$ & Woods & 1294 & & & Lettuce-romaine-4 week & 1068 \\
\hline $\mathrm{C} 12$ & Bldg-grass-trees-drives & 380 & & & Lettuce-romaine-5 week & 1927 \\
\hline $\mathrm{C} 13$ & & & & & Lettuce-romaine-6 week & 916 \\
\hline C14 & & & & & Lettuce-romaine-7 week & 1070 \\
\hline $\mathrm{C} 15$ & & & & & Vineyard-untrained & 7268 \\
\hline \multirow[t]{2}{*}{$\mathrm{C} 16$} & & & & & Vineyard-vertical-trellis & 1807 \\
\hline & Total & 10171 & Total & 43923 & Total & 54129 \\
\hline
\end{tabular}

Figure 1: INFORMATION OF THE THREE CONSIDERED DATA SETS.

\subsection{Experimental setup}

To evaluate the performance of the proposed algorithms, we design four different strategies for the task

235 of-the-art multi-kernel methods.

1) SK-CV (RBF): A SVM with a single kernel and adopting the RBF kernel as the kernel function in the SVM, whose parameter $\gamma$ is determined by cross-validation (CV).

2) SK-POLY: Standard SVM with a single kernel and adopting a polynomial kernel as the kernel function 240 in the SVM.

3) Mahal method (round-1): We chose all the training samples to learn the transformation matrix with Algorithm 2 and obtain the transformed matrix $M 1$. The parameter of the kernel is determined by CV.

4) Mahal method (round-2-I): Based on 3), we repeat the method of 3) and choose all samples of all 


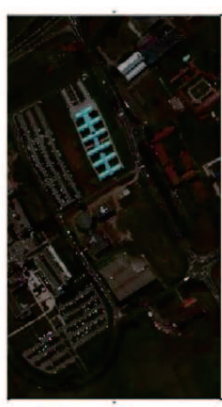

False color map

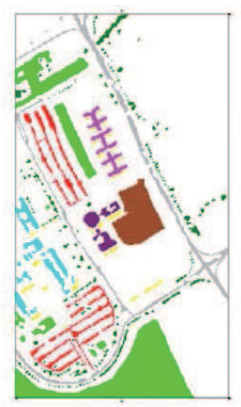

Truthmap

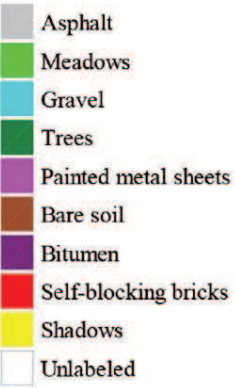

Unlabeled

(a) Ground-truth map of the Pavia University data set (9 (b) Ground-truth map of the Salinas data set (16 land cover classland cover classes).

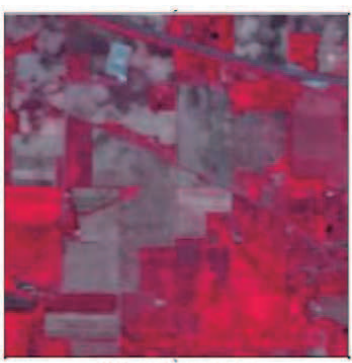

False color map

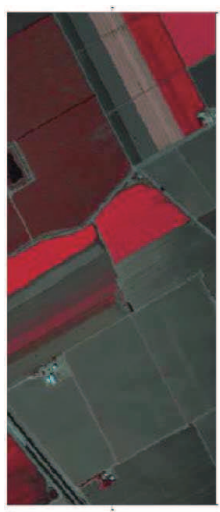

False color map

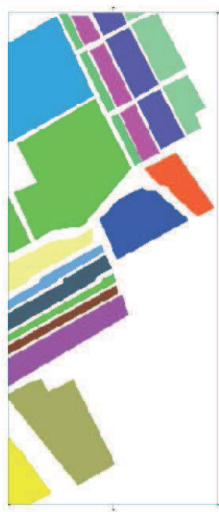

Truthmap
Broccoli-green-weeds, Broccoli-green-weeds Fallow

Fallow-rough-plow

Fallow-smooth

Stubble

Celery

Grapes-untrained

Soil-vinyary-develop

Corn-senesced-green-weeds

Lettuce-romaine-4 week

Lettuce-romaine- 5 week

Lettuce-romaine- 6 week

Lettuce-romaine-7 week

Vineyard-untrained

Vineyard-vertical-trellis

Unlabeled

es).

(c) Ground-truth map of the Indian Pines data set (12 land cover classes).

Figure 2: Ground-truth map of several hyperspectral images 
classes to learn the transformation matrix $M 2$ with Algorithm 2 in the second iteration. The parameter of combines multiple kernels with NMF.

8) KNMF-MKL: The kernel-based nonnegative matrix factorization (KNMF) MKL method, also proposed by Gu and Wang(2015)[18, which combines multiple kernels with the KNMF method.

Fig. $\{3\}$ depicts the framework of strategies 3)-6) proposed above.

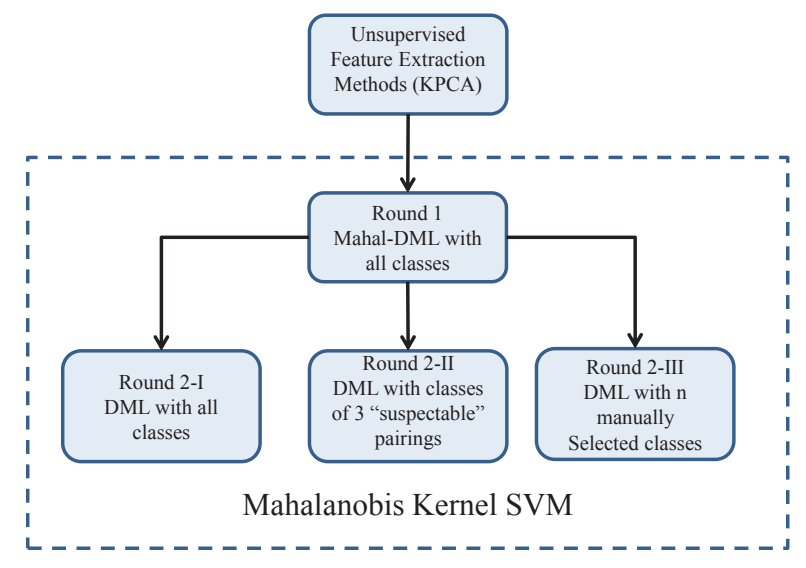

Figure 3: The strategies of the experimental designs

5) Mahal method (round-2-II): We learn a transformation matrix $M 2$ with Algorithm3 where we set $n=3$. The parameter of the kernel is determined by CV.

6) Mahal method (round-2-III): Based on 3), we select samples of several classes via experiments to learn the transformation matrix M2 with Algorithm 2 The parameter of the kernel is determined by CV.

7) NMF-MKL: The nonnegative matrix factorization (NMF) MKL proposed by Gu and Wang[18, which

In [53], Gu adopted KPCA in the feature extraction step of HSI classification. Their paper proves that, using the kernel trick, the capability of the PCA feature extraction can be improved. Therefore, for all the experiments, we adopted KPCA as the feature extraction method. In this paper, we adopt a polynomial kernel as the kernel function in KPCA, and for the Indiana Pines data set, the parameters of the kernel were tuned to $c=6, d=4$; we also extracted the first 20 components for classification. For the Pavia University and Salinas Valley data sets, the parameters of the kernel were tuned to $c=5, d=3$, and the first 30 components were extracted.

For method 2), the parameter of the polynomial kernel was also tuned by experiment, and for other RBFbased SVMs, all parameters were determined by cross validation. For NMF-MKL and KNMF-MKL[18, considering the hardware configuration, we chose 20 RBF kernels as the basic kernel, and the band width $\sigma$ ranged from 0.1 to 2 , with a step size of 0.1. For KNMF, following, we used the polynomial kernel function 
to identify the weights of the selected basic kernels:

$$
k\left(k_{i}, k_{j}\right)=\left(k_{i} * k_{j}+1\right)^{6}
$$

To evaluate the performances of the proposed methods under the condition of the high dimensionality of images with quite insufficient a prior information, the numbers of labeled samples for training ranged from 10 to 50 for each class in every data set, which are substantially smaller than the numbers of spectral bands

of these hyperspectral images. For each experiment, the classification result is an average of ten Monte Carlo runs used to generate a general classification accuracy.

In this paper, the overall accuracy (OA), average accuracy (AA) and kappa coefficient $(\kappa)$ [54] are utilized, where OA denotes the number of correctly classified samples divided by the total number of test samples and AA denotes the average of the individual class accuracies. $\kappa$ involves both omission and commission errors and is thus a more robust measure compared to OA and AA. They are formulated as follows:

$$
\begin{gathered}
O A=\frac{n_{A}}{N_{A}}, A A=\frac{1}{C} \sum_{i=1}^{C} \frac{n_{i}}{N_{i}} \\
\kappa=\frac{P_{0}-P_{e}}{1-P_{e}}
\end{gathered}
$$

In Eq. (28), $n_{A}$ and $N_{A}$ denote the total number of correctly classified samples and the total number of test samples, $n_{i}$ and $N_{i}$ denote the number of correctly classified samples and the number of test samples in class $i$, and $C$ is the number of classified classes [18]. For Eq. 29], $P_{0}=\sum_{i}^{C} P_{i i}=\sum_{i}^{C} \frac{M_{i i}}{\tilde{N}}$ is the "observed" agreement, $C$ is the number of classes, $M_{i i}$ is the $i$ th diagonal element of the confusion matrix, $\tilde{N}$ is the total number of test samples, $P_{e}=\sum_{i}^{C}\left(P_{i .} P_{. i}\right)$ is the "expected" agreement, $P_{i}=\frac{\tilde{R}_{i}}{\tilde{N}}$, and $P_{. i}=\frac{\tilde{C}_{i}}{\tilde{N}}$, where $\tilde{R}_{i}$ and $\tilde{C}_{i}$ denote the sum of the $i$ th row and column, respectively, of the confusion matrix.

Substituting $P_{o}$ and $P_{e}$ into Eq. 29 yields

$$
\kappa=\frac{\sum_{i}^{C} P_{i i}-\sum_{i}^{c}\left(P_{i .} P_{. i}\right)}{1-\sum_{i}^{C}\left(P_{i .} P_{. i}\right)}=\frac{\tilde{N} \sum_{i}^{C} M_{i i}-\sum_{i}^{C} \tilde{R_{i}} \tilde{C_{i}}}{\tilde{N}^{2}-\sum_{i}^{C} R_{i} \tilde{C}_{i}}
$$

\subsection{Results and Analysis}

1) AVIRIS Indiana Pines Dataset: Fig, 4 shows the classification results obtained by different algorithms. As mentioned above, in this paper, our method attempts to improve several classes that are difficult to identify by common methods. This means that we will pay greater attention to the classification accuracy on a per-class basis compared to the total accuracy; therefore, our first goal is to compare the AA with varying numbers of training samples per class. Fig $4(\mathrm{a})$ shows that, with increasing number of training samples, the AA increases under all methods following a similar trend. In addition, with increasing number of training 


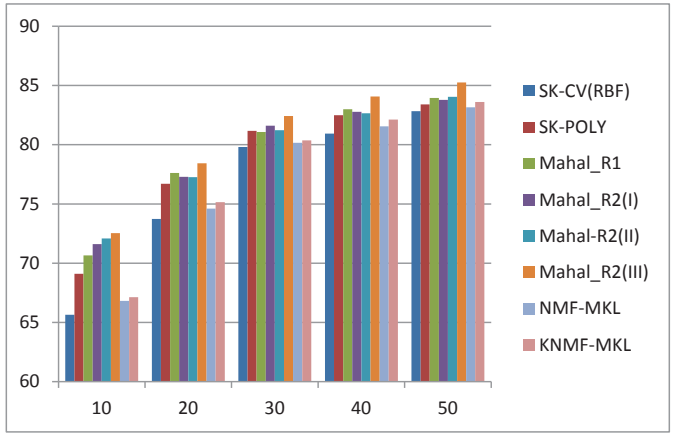

(a) AA in percentage for the eight methods on the AVIRIS Indian Pines data set.

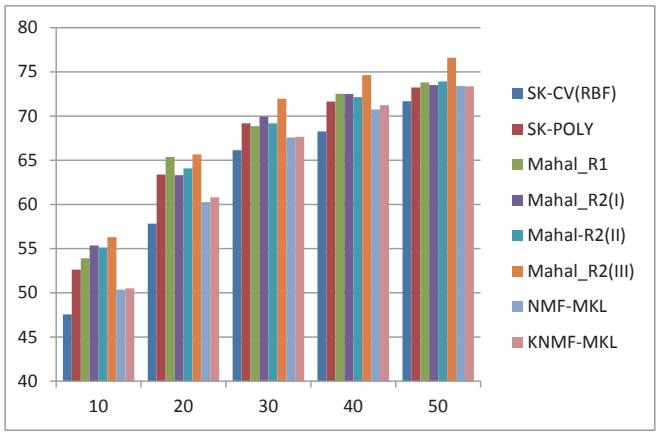

(b) AA of 6 classes in percentage for the eight methods on the AVIRIS Indian Pines data set.

Figure 4: AA of the AVIRIS Indiana Pines data set

samples, the difference between the methods becomes smaller. Generally, the SK-CV performs the worst out of all methods, and the two MKL methods perform better than the SK-CV. This result is similar to the result obtained in [18]. In addition, the Mahal-R2(III) method performs better than the other methods under all conditions, especially under the extreme condition (i.e., the number of samples per class is no greater than 20). Moreover, the other strategies proposed in this paper also perform better than the other methods. Further, what we want to emphasize is that, for this dataset, SK-Poly's method performed better than SK-CV and the multi-kernel methods under most conditions.

Table 1: Results obtained using different methods for the Indian Pines data set, with 20 labeled samples per class

\begin{tabular}{|c|c|c|c|c|c|c|c|c|}
\hline Class & SK-CV(RBF) & SK-POLY & Mahal R1 & Mahal R2(I) & Mahal R2(II) & Mahal R2(III) & NMF-MKL & KNMF-MKL \\
\hline \hline$\sigma_{1}$ & 54.38 & 64.87 & 64.97 & 61.27 & 61.87 & $\mathbf{6 5 . 5 2}$ & 60.30 & 60.28 \\
\hline$\sigma_{2}$ & 57.74 & $\mathbf{6 5 . 7 0}$ & 65.04 & 63.32 & 64.58 & 61.29 & 62.60 & 62.80 \\
\hline$\sigma_{3}$ & 73.55 & 74.77 & 73.88 & 76.12 & 78.36 & $\mathbf{8 1 . 0 3}$ & 71.59 & 72.38 \\
\hline$\sigma_{4}$ & 90.13 & 91.15 & 88.89 & 90.08 & $\mathbf{9 1 . 2 2}$ & 89.98 & 90.36 & 90.31 \\
\hline$\sigma_{5}$ & 89.56 & 90.52 & 90.91 & 91.87 & 92.31 & $\mathbf{9 2 . 7 0}$ & 89.38 & 91.05 \\
\hline$\sigma_{6}$ & 98.23 & 99.04 & 99.30 & 99.19 & $\mathbf{9 9 . 3 6}$ & 99.30 & 98.32 & 98.49 \\
\hline$\sigma_{7}$ & 68.05 & 66.19 & 71.61 & 72.31 & 70.62 & $\mathbf{7 1 . 9 3}$ & 68.29 & 68.38 \\
\hline$\sigma_{8}$ & 55.86 & 53.76 & 56.89 & 57.25 & 53.58 & $\mathbf{5 8 . 0 4}$ & 52.75 & 54.41 \\
\hline$\sigma_{9}$ & 57.73 & 71.08 & 74.39 & 72.27 & 69.44 & $\mathbf{7 5 . 8 1}$ & 60.64 & 62.04 \\
\hline$\sigma_{10}$ & 99.06 & 99.27 & 99.01 & $\mathbf{9 9 . 4 8}$ & 99.11 & 99.43 & 98.96 & 99.11 \\
\hline$\sigma_{11}$ & $\mathbf{8 7 . 3 0}$ & 85.31 & 87.22 & 85.80 & 87.16 & 84.87 & 84.89 & 85.66 \\
\hline$\sigma_{12}$ & 53.22 & 58.69 & 59.28 & 58.11 & 59.83 & $\mathbf{6 1 . 3 1}$ & 57.08 & 56.94 \\
\hline AA (\%) & 73.73 & 76.70 & 77.62 & 77.26 & 77.29 & $\mathbf{7 8 . 4 3}$ & 74.60 & 75.15 \\
\hline OA(\%) & 67.03 & 69.37 & 70.95 & 70.29 & 69.63 & $\mathbf{7 1 . 2 4}$ & 67.48 & 68.22 \\
\hline$\kappa(\%)$ & 64.23 & 66.96 & 68.75 & 67.99 & 67.27 & $\mathbf{6 9 . 0 6}$ & 64.81 & 65.63 \\
\hline
\end{tabular}

Table 1 shows the AA of each individual class for 10 trials. The OA, AA and kappa coefficient of the eight 


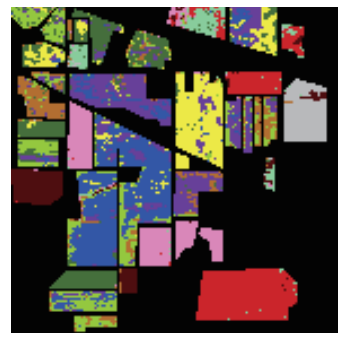

(a) SK-RBF kernel.

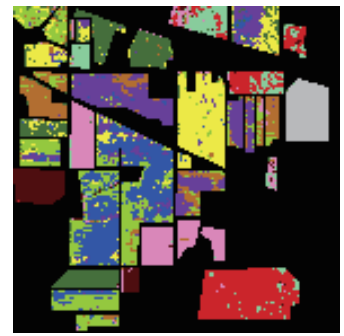

(e) Mahal-kernel R2(II).

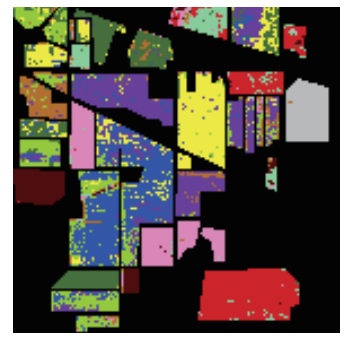

(b) SK-Polynomial kernel.

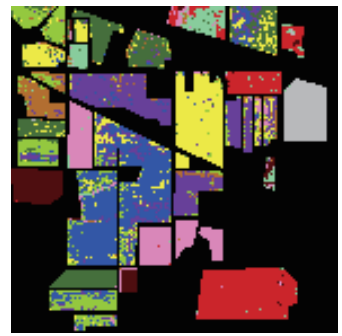

(f) Mahal-kernel R2(III).

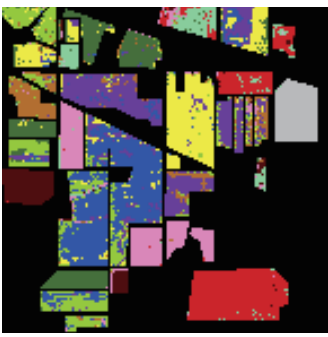

(c) Mahal-kernel R1.

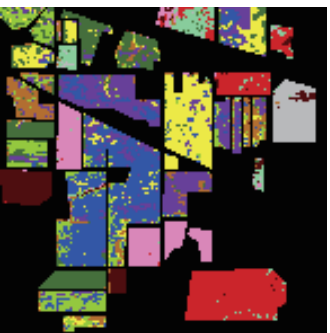

(g) NMF kernel.

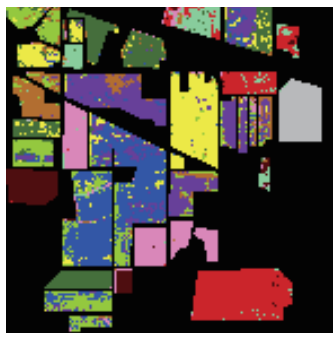

(d) Mahal-kernel R2(I).

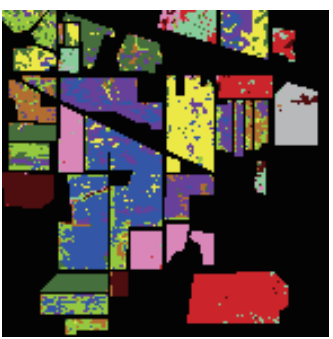

(h) KNMF kernel.

Figure 5: Classification maps for various methods on the Indiana Pines data set

that, compared with SK-CV (which is the most similar to the methods proposed in this paper), our method obviously performs better for Corn-notill (C1), Corn-mintill (C2), Soybean-notill (C7), Soybean-mintill (C8), Soybean-mintill (C9), and Building-Grass-Trees-Drives (C12); for the other classes, the proposed methods also perform similarly or even better than the compared methods. Therefore, our methods perform the best in terms of the AA, OA and kappa coefficient.

To highlight our methods' abilities to improve the accuracy for the hard-to-identify classes, we compared the AA of the six worst performing classes $(\mathrm{C} 1, \mathrm{C} 2, \mathrm{C} 7, \mathrm{C} 8, \mathrm{C} 9$ and $\mathrm{C} 12$, where the test samples of these classes make up more than 65 percent of all the samples). From Fig 4(b), we can see that the performance of the proposed methods is similar to the AA of all the classes. This shows that our methods improved the classification of these hard-to-identify classes. In this figure, we can see that SK-Poly performs better than all the other comparison methods.

2) ORSIS Pavia University Dataset: Table 2 shows the AA of each individual class for 10 trials. The OA, AA and Kappa coefficient of the eight methods were obtained using 20 randomly chosen labeled samples for each class. We can see that the Mahal-R2(II) and Mahal-R2(III) demonstrated the best overall performances and also obtained the best performance in terms of all three coefficients. Identically to the above, we pick Asphalt (C1), Meadows (C2), Gravels (C3), Bare Soil (C6), and Self-blocking bricks (C8) (the test samples for these classes constitute approximately 41 percent of all the samples) as the "hard-to-identify" classes to observe. The results show that Mahal-R2(II) performs best in terms of OA and Kappa, whereas MahalR2(III)'s AA is the best out of all methods. Moreover, the performances of both Mahal-R2(I) and MahalR2(II) are not quite steady, and Mahal-R2(I) performs poorly in C2. Meanwhile, Mahal-R2(II) performs 


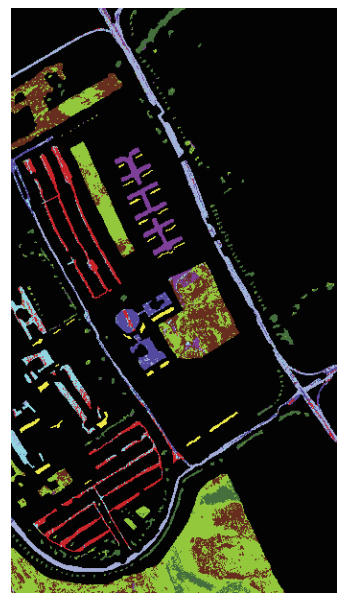

(a) SK-RBF kernel.

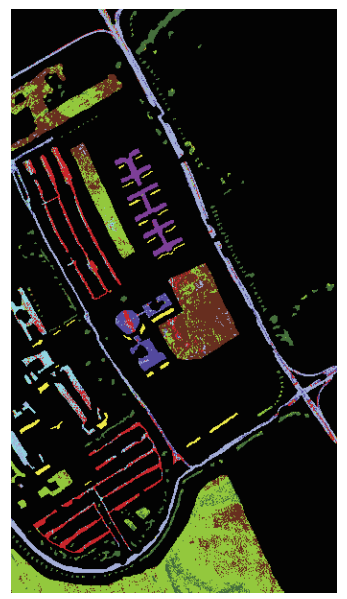

(e) Mahal-kernel R2(II).

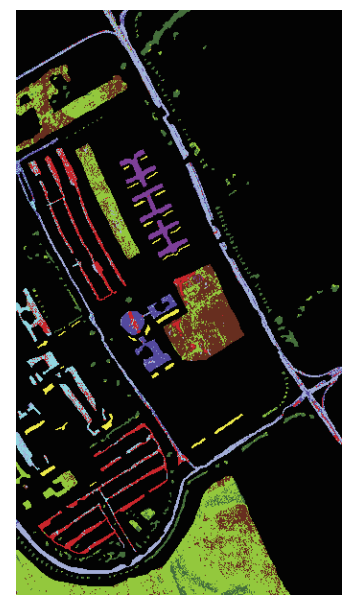

(b) SK-Polynomial kernel.

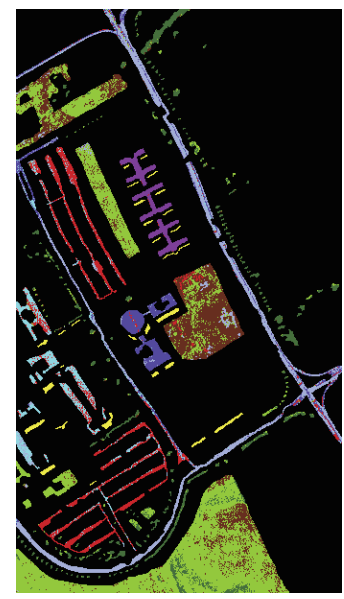

(f) Mahal-kernel R2(III)

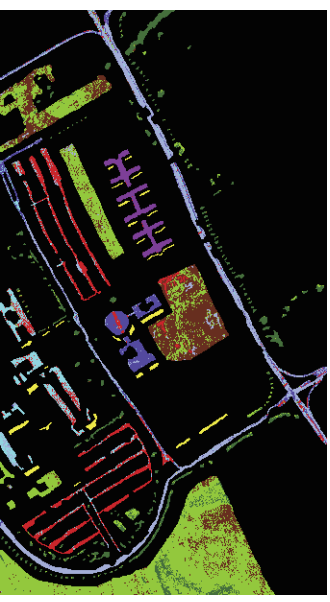

(c) Mahal-kernel R1.

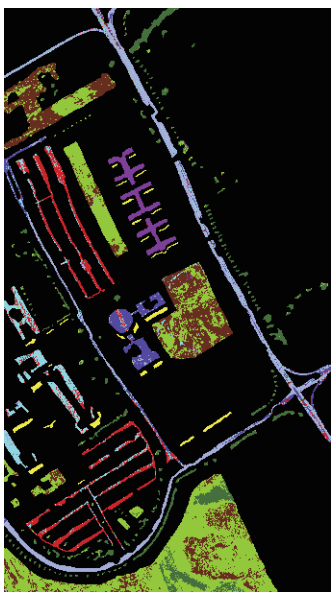

(g) NMF kernel.

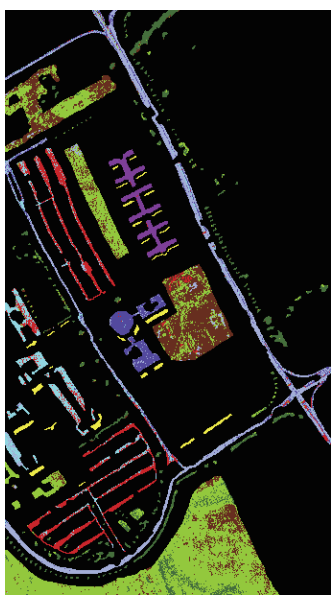

(d) Mahal-kernel R2(I).

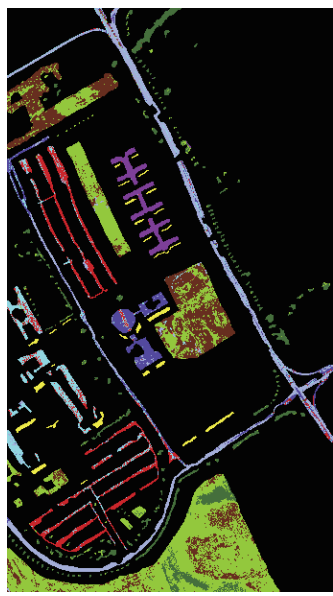

(h) KNMF kernel.

Figure 6: Classification maps for various methods on the Pavia University data set

Table 2: Results for different methods on the Pavia University data set, with 20 labeled samples per class

\begin{tabular}{|c|c|c|c|c|c|c|c|c|}
\hline Class & SK-CV(RBF) & SK-POLY & Mahal R1 & Mahal R2(I) & Mahal R2(II) & Mahal R2(III) & NMF-MKL & KNMF-MKL \\
\hline \hline$\sigma_{1}$ & 75.57 & 71.73 & 72.77 & 72.11 & $\mathbf{7 6 . 8 2}$ & 72.76 & 73.13 & 73.99 \\
\hline$\sigma_{2}$ & $\mathbf{7 5 . 5 2}$ & 71.55 & 66.03 & 71.29 & 75.49 & 74.37 & 62.75 & 64.92 \\
\hline$\sigma_{3}$ & $\mathbf{8 0 . 2 8}$ & 78.08 & 77.75 & 65.08 & 79.33 & 74.72 & 75.98 & 77.12 \\
\hline$\sigma_{4}$ & 85.82 & 84.84 & 83.33 & 84.09 & 86.3 & $\mathbf{8 6 . 4 9}$ & 84.66 & 84.35 \\
\hline$\sigma_{5}$ & 99.25 & 99.47 & $\mathbf{9 9 . 7}$ & 99.67 & 99.65 & 99.67 & 99.62 & 99.47 \\
\hline$\sigma_{6}$ & 74.84 & 75.24 & $\mathbf{7 7 . 2 3}$ & 74.75 & 74.56 & 75.42 & 73.79 & 74.71 \\
\hline$\sigma_{7}$ & 85.50 & 88.24 & $\mathbf{9 2 . 1 9}$ & 91.78 & 87.94 & 91.65 & 86.23 & 86.39 \\
\hline$\sigma_{8}$ & 71.22 & 76.54 & 77.64 & $\mathbf{8 4 . 1 5}$ & 75.52 & 82.17 & 74.76 & 74.69 \\
\hline$\sigma_{9}$ & $\mathbf{9 9 . 9 3}$ & 99.86 & 99.53 & 99.46 & 99.82 & 99.89 & 99.68 & 99.75 \\
\hline AA $(\%)$ & 82.8408 & 83.1013 & 82.9067 & 82.4873 & 83.9368 & $\mathbf{8 4 . 1 2 7}$ & 81.1781 & 81.7091 \\
\hline OA $(\%)$ & 75.7098 & 77.6207 & 73.78 & 75.6683 & $\mathbf{7 8 . 2 1 3 9}$ & 77.6724 & 71.5748 & 72.7964 \\
\hline$\kappa(\%)$ & 69.1433 & 71.3706 & 67.0206 & 69.1846 & $\mathbf{7 2 . 1 2 9 3}$ & 71.5204 & 64.4206 & 65.811 \\
\hline
\end{tabular}



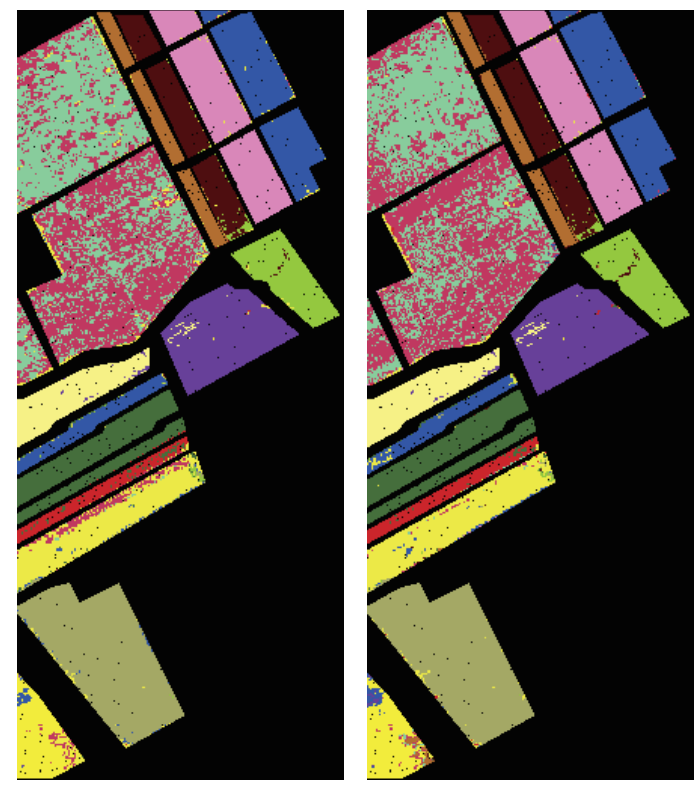

(a) SK-RBF kernel.

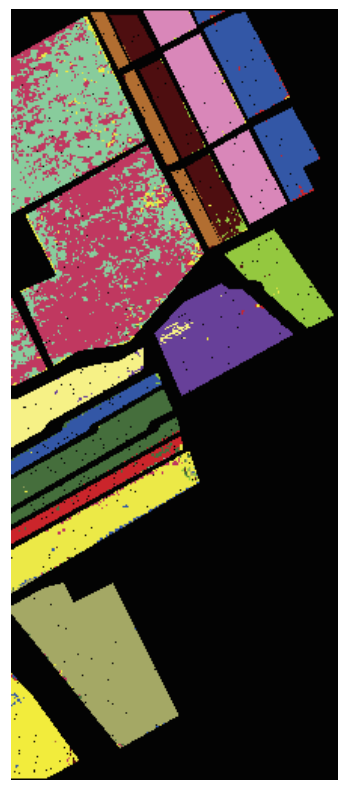

(e) Mahal-kernel R2(II). (b) SK-Polynomial kernel.

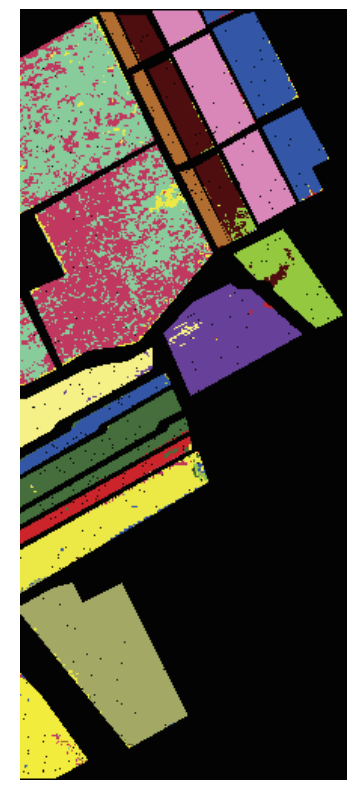

(f) Mahal-kernel R2(III)

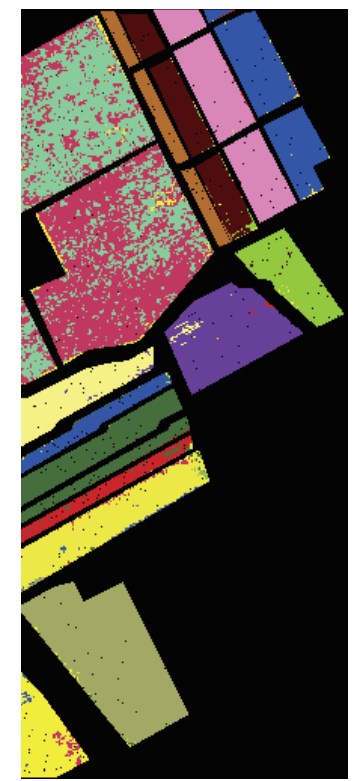

(c) Mahal-kernel R1.

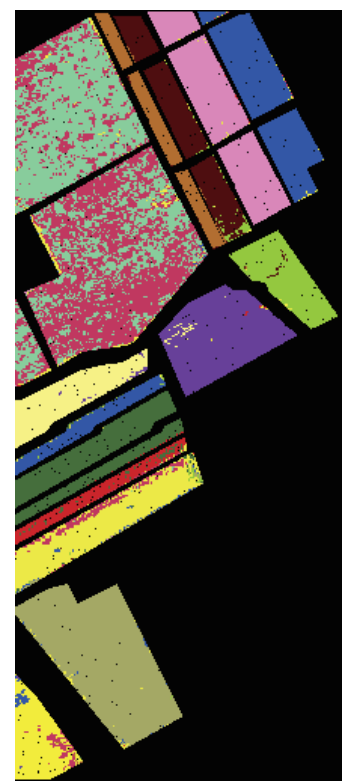

(g) NMF kernel.

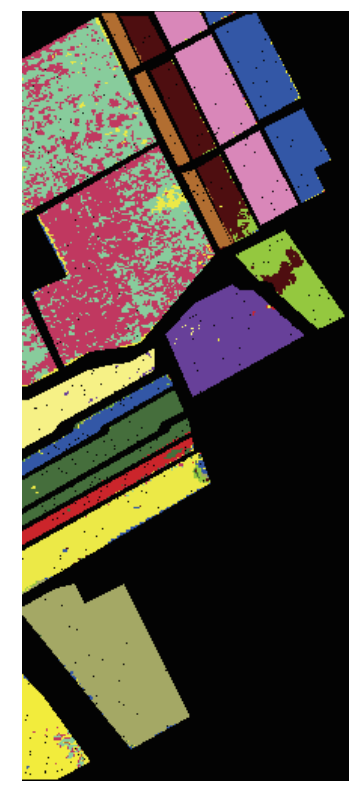

(d) Mahal-kernel R2(I).

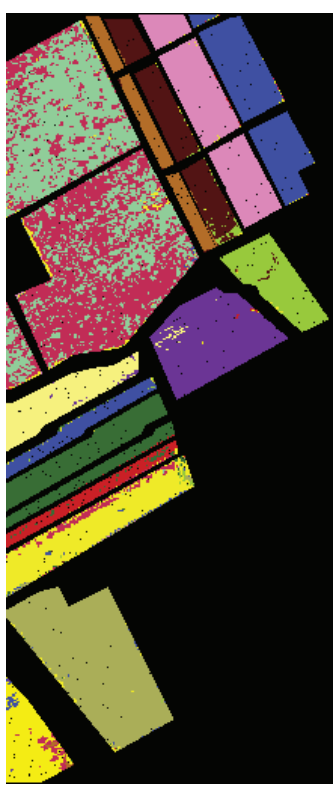

(h) KNMF kernel.

Figure 7: Classification maps for various methods on the Salinas data set 


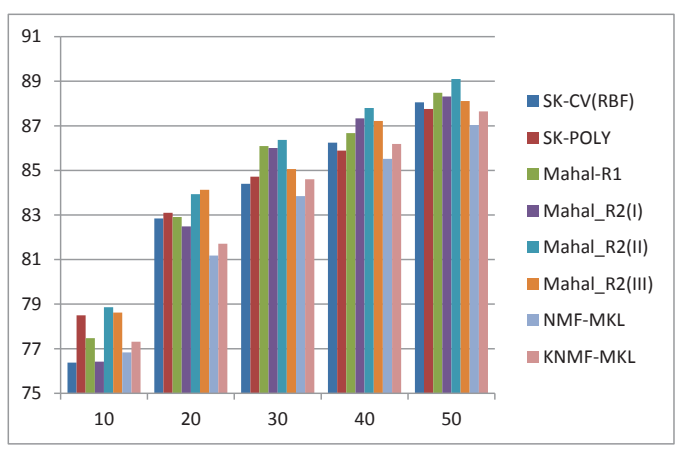

(a) AA in percentage for the eight methods for the ROSIS Pavia University data set

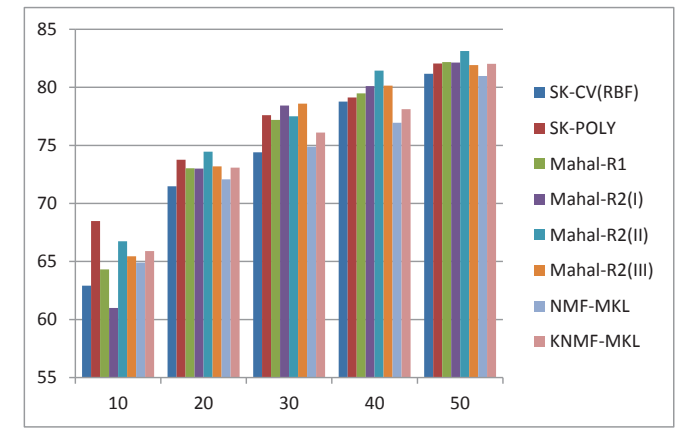

(b) AA of 5 classes in percentage for the eight methods for the ROSIS Pavia University data set.

Figure 8: AA in percentage for the ROSIS Pavia University data set

poorly compared to most methods in $\mathrm{C} 7 \& \mathrm{C} 8$, which hinder their performances. Mahal-R2(III) performs the best in only two insignificant classes (C4 \& C5, which only constitute approximately $10 \%$ of all samples, and the improvement is limited); however, for other classes, its performance is also outstanding and stable; therefore, it obtains the highest AA of all methods.

Fig 8 shows the AA of eight methods for all classes and the selected classes with different sizes of training samples. Fig 8(a) depicts that, when the number of training samples ranges from 10 to 50, at least one of Mahal-R2(II) and Mahal-R2(III) performs the best out of all methods. In addition, the accuracy of Mahal-R1 and Mahal-R2(I) improves, and when the number of samples is greater than 30, their performances are similar to or even better than Mahal-R2(III).From Fig $8(\mathrm{~b})$, we can see that the trend of the results for the hard-toidentify classes is generally similar to that of the AA of all classes. On most occasions, the performance of Mahal-R2(II) and Mahal-R2(II) is the highest. In addition, this figure shows that the performance of Mahal$\mathrm{R} 2$ (III) is better than the AA of all classes. This means that its ability to distinguish the close pairwise classes is more significant.

In this data set, the performances of SK-CV and SK-Polys differ with increasing number of training samples. When the number of training samples is less than 30, the SK-Poly obtains an excellent performance. Fig $8(\mathrm{a})$ shows the results when the samples size is 10. SK-Poly's performance is the best of all the methods with the hard-to-identify classes and obtains the three highest performances with all classes. In contrast, SK-CV's performance is poor; however, as the number of samples increases, SK-CV surpasses SK-Poly. On the other hand, NMF-MKL and KNMF-MKL perform moderately when the number of training samples is greater more than 30. They perform slightly better than does SK-CV; however, when the number of samples increases, this advantage gradually diminishes.

3) AVIRIS Salinas Valley Data set: From Table 3 , we can see the accuracy on a per-class basis for the AA, OA and Kappa coefficient. Among the methods, three AAs of the Mahal-R2 methods are over 93\%, and Mahal-R2(II)'s AA, OA and Kappa are as high as $94.01 \%, 88.04 \%$ and $86.71 \%$, respectively. For this data set, we chose Grapes-untrained(C8), Corn-senesced-green-seed(C10) and Vineyard-untrained(C15) as the hard- 
to-identify classes (the test samples of these classes constitute approximately 40.3 percent of the samples). Compared with SK-CV, both Mahal-R2(I) and Mahal-R2(II) obtained a higher accuracy with C8 and C10; however, the accuracy with C15 is less than that for SK-CV and SK-Poly. SK-Poly achieves the highest accuracy with C15 (71.21\%); however, its accuracy with C8 (58.97\%) is almost the worst of all methods. Therefore, Mahal-R2(II) performs the best among all methods in terms of the AA for hard-to-identify classes.

Fig 9 shows the AA of eight methods for all classes when the number of training samples ranges from 10 to 50. We can see that, when the number of training samples is not sufficient (no more than 30), MahalR2(II) performs much better than all the other methods. However, with increasing sample size, the results become similar, although Mahal-R2(II) still performs better than the other methods. Concerning the other methods, the advantage of SK-Poly's result is no longer as good as the other two data sets mentioned above. Its performance is similar to that of SK-CV and slightly better than those of the two MKL methods when the sample size is no greater than 30 .

Table 3: Results for different methods on the Salinas Valley data, with 20 labeled samples per class

\begin{tabular}{|c|c|c|c|c|c|c|c|c|}
\hline Class & SK-CV(RBF) & SK-POLY & Mahal R1 & Mahal R2(I) & Mahal R2(II) & Mahal R2(III) & NMF-MKL & KNMF-MKL \\
\hline \hline$\sigma_{1}$ & 98.05 & 96.77 & 97.28 & 97.63 & 98.22 & $\mathbf{9 8 . 3 5}$ & 97.74 & 97.76 \\
\hline$\sigma_{2}$ & 98.08 & 98.35 & 97.99 & 98.54 & $\mathbf{9 9 . 5 3}$ & 98.97 & 97.86 & 98.14 \\
\hline$\sigma_{3}$ & 94.91 & 92.39 & 94.11 & 96.13 & $\mathbf{9 6 . 1 8}$ & 92.65 & 92.91 & 92.33 \\
\hline$\sigma_{4}$ & 99.36 & 99.24 & $\mathbf{9 9 . 6 1}$ & 99.45 & 99.51 & 99.51 & 99.29 & 99.29 \\
\hline$\sigma_{5}$ & 93.24 & 94.47 & 96.01 & 96.09 & $\mathbf{9 7 . 1 2}$ & 97.1 & 94.12 & 94.43 \\
\hline$\sigma_{6}$ & 96.94 & 97.95 & 98.89 & 98.24 & $\mathbf{9 9 . 3 6}$ & 98.47 & 98.16 & 98.42 \\
\hline$\sigma_{7}$ & 98.82 & 98.93 & 98.72 & 98.97 & $\mathbf{9 9 . 2}$ & 99.09 & 98.76 & 98.85 \\
\hline$\sigma_{8}$ & 61.64 & 58.97 & 67.56 & 66.14 & $\mathbf{7 0 . 5}$ & 60.63 & 58.92 & 62.11 \\
\hline$\sigma_{9}$ & 96.29 & 96.52 & 97.11 & 97.76 & 98.26 & $\mathbf{9 8 . 3 4}$ & 96.16 & 96.29 \\
\hline$\sigma_{10}$ & 84.9 & 88.73 & 85.9 & 87.77 & $\mathbf{9 2 . 0 1}$ & 89.48 & 83.25 & 83.5 \\
\hline$\sigma_{11}$ & 91.34 & 91.53 & 92.86 & 93.23 & $\mathbf{9 4 . 4 3}$ & 92.86 & 91.77 & 91.97 \\
\hline$\sigma_{12}$ & 97.73 & 98.00 & 99.69 & 99.71 & 99.69 & $\mathbf{9 9 . 7 8}$ & 97.71 & 97.77 \\
\hline$\sigma_{13}$ & 97.83 & 97.79 & 98.42 & 98.37 & $\mathbf{9 9 . 2 4}$ & 98.55 & 96.76 & 97.12 \\
\hline$\sigma_{14}$ & 91.81 & 93.03 & 91.83 & 92.88 & $\mathbf{9 5 . 4 7}$ & 92.36 & 90.00 & 90.19 \\
\hline$\sigma_{15}$ & 70.67 & $\mathbf{7 1 . 2 1}$ & 60.9 & 65.01 & 67.99 & 66.54 & 66.6 & 67.51 \\
\hline$\sigma_{16}$ & 95.19 & 94.28 & 96.31 & 95.33 & $\mathbf{9 7 . 4 6}$ & 97.35 & 93.73 & 94.34 \\
\hline AA $(\%)$ & 91.67 & 91.76 & 92.07 & 92.58 & $\mathbf{9 4 . 0 1}$ & 92.5 & 90.86 & 91.25 \\
\hline OA $(\%)$ & 85.04 & 84.84 & 85.48 & 86.01 & $\mathbf{8 8 . 0 4}$ & 85.3 & 83.74 & 84.64 \\
\hline$\kappa(\%)$ & 83.43 & 83.2 & 83.87 & 84.47 & $\mathbf{8 6 . 7 1}$ & 83.7 & 81.99 & 82.97 \\
\hline
\end{tabular}

The AAs of the three hard-to-identify classes shown in Fig 9(b) are quite similar to the total AA. As was the case for the experiment on the Pavia University data set, SK-Poly obtains an excellent performance when the samples are quite insufficient. However, as the sample size increases, this performance advantage becomes less obvious.

4) Analysis of Kappa coefficient and computational cost.

The Kappa test [54] and computational cost are compared in a later section. The results of the Kappa 


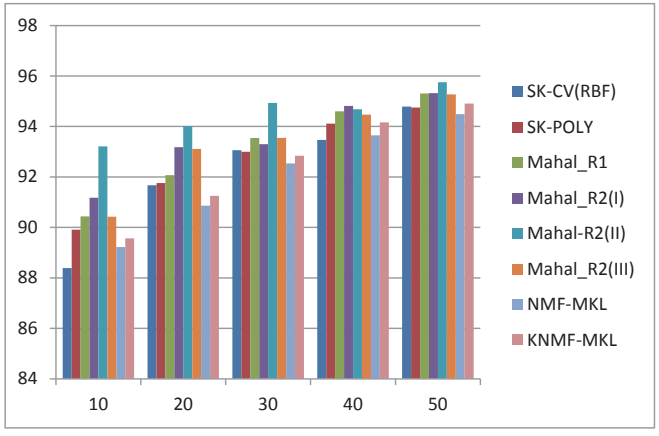

(a) AA in percentage for the eight methods on the AVIRIS Salinas data set.

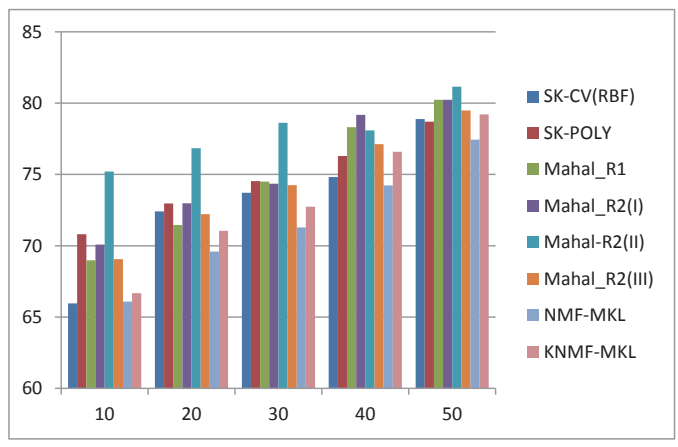

(b) AA of 3 classes in percentage for the eight methods on the AVIRIS Salinas data set.

Figure 9: AA in percentage for the AVIRIS Salinas data set.
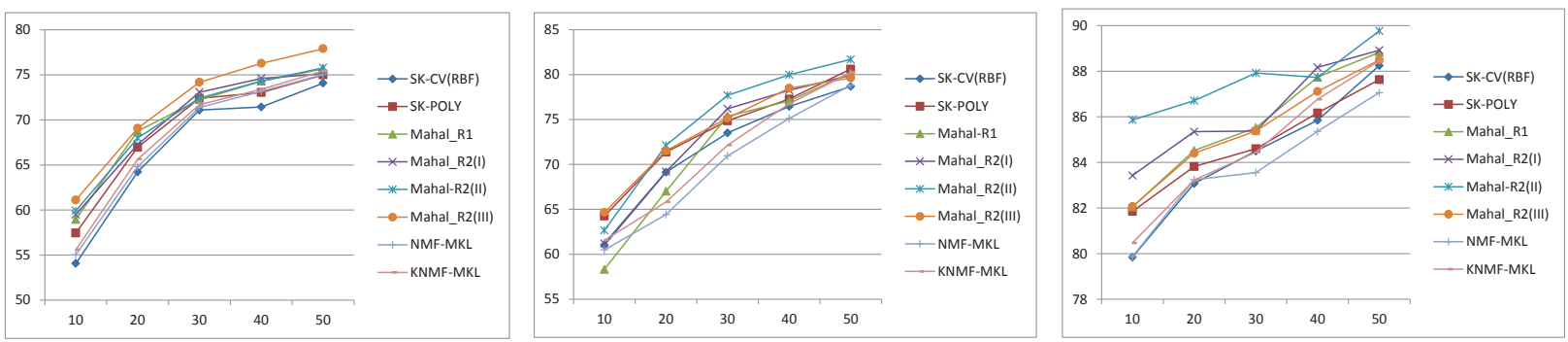

(a) Kappa in percentage for the eight (b) Kappa in percentage for the eight (c) Kappa in percentage for the eight methods on the AVIRIS Indian Pines data methods on the ROSIS Pavia University methods on the AVIRIS Salinas Valley daset.

data set.

ta set.

Figure 10: Kappa coefficient in percentage for the eight methods on 3 hyperspectral images 

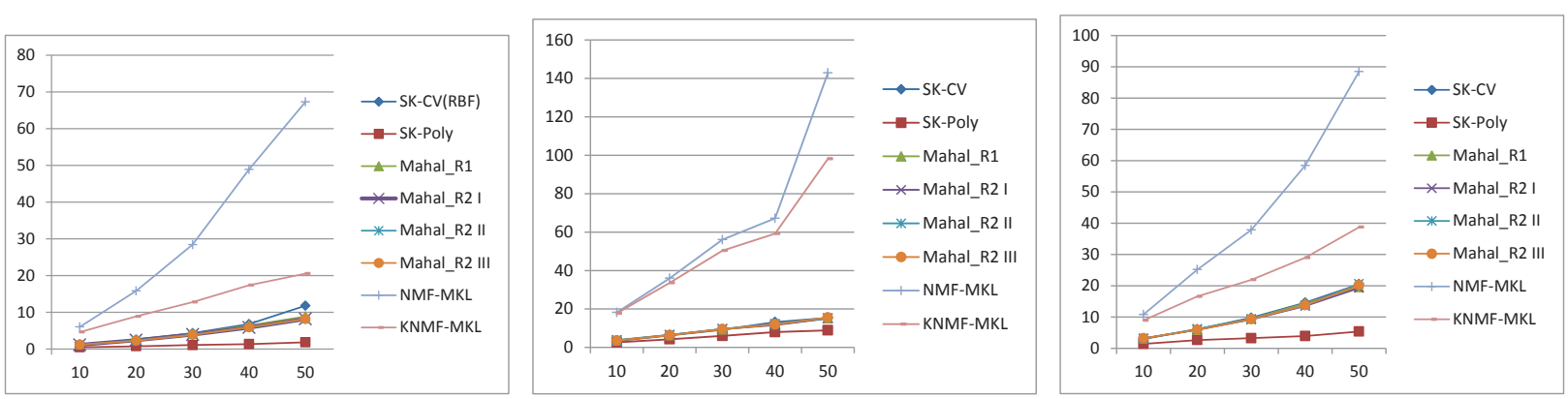

(a) Computing cost of the eight methods (b) Computing cost of the eight methods (c) Computing cost of the eight methods on the AVIRIS Indian Pines data set. on the ROSIS Pavia University data set. on the AVIRIS Salinas Valley data set.

Figure 11: Computational cost for 3 hyperspectral images

test are shown in Table 10 .

The $\kappa$ coefficient is computed as $\frac{P(A)-P(E)}{1-P(E)} . P(A)$ is the observed agreement among the coders, and $P(E)$ is the expected agreement, that is, $P(E)$ represents the probability that the coders agree by chance. The values of $\kappa$ are constrained to the interval $[-1,1] . \kappa=1$ means perfect agreement, $\kappa=0$ means that agreement is equal to chance, and $\kappa=-1$ means perfect disagreement.

With the Kappa test results, we can conclude that Mahal-based methods performed significantly better compared to other methods. The best performance on every data set is obtained by the proposed Mahal-based methods. The trend exhibited by these results is similar to the trends followed by the AA and OA mentioned above. For example, Mahal-R2(III) scores the highest under all conditions on the Indiana Pines data set, Mahal-R(II) and Mahal-R2(III) obtain the highest performances on the PaviaU data set, and Mahal-R2(II) performs the best on the Salinas data set.

Comparing the computational time, Fig.11 shows the computational times on the three data sets. The computational cost was recorded with a $\mathrm{PC}$ with a $2.6 \mathrm{GHz}$ i5-3320 processor and 4GB RAM. All the results with standard deviations are averages of 10 repeated experiments. From the figures, we can find that SKPOLY obtains the lowest computational cost, which can be easily understood because it omits the parameter optimization process. Both KNMF-MKL and NMF-MKL adopt non-negative matrix factorization to optimize the kernel weights, although the latter requires more time because it need more memory to save the kernel matrix and has more dimensions to compute. In our experiments, when computing on a larger data set (i.e., the Pavia University data set) and when the sample size is large (i.e., the size is tuned to 50), the hardware, especially the memory, becomes the bottleneck of the computing efficiency. This explains the fact that, when the training size increases, the curve obtained using NMF-MKL cannot remain linear with increasing sample size on some data sets. We want to emphasize the performance of our proposed methods. The three figures show that the computational costs of these four Mahalanobis DML-based methods are quite similar to that of the SK-CV, which means that the process of Mahalanobis DML is relatively computational inexpensive compared with the total cost. 


\section{Conclusion}

\section{Acknowledgment}

This work is supported by the Program for New Century Excellent Talents in University under Grant No. NCET-13-0168 and the National Science Foundation of China under Grant No.61371178. 


\section{References}

[1] S. T. Nahr, P. Pahlavani, M. Hasanlou, Different optimal band selection of hyperspectral images using a continuous genetic algorithm, The International Archives of Photogrammetry, Remote Sensing and Spatial Information Sciences 40 (2) (2014) 249.

[2] G. P. Hughes, On the mean accuracy of statistical pattern recognizers, in: IEEE Trans. Inf. Theory 1968, 1968, pp. 55-63.

[3] B. E. Boser, I. M. Guyon, V. N. Vapnik, A training algorithm for optimal margin classifiers, Proceedings of Annual Acm Workshop on Computational Learning Theory 5 (1996) 144-152.

[4] C. J, C burges 1998 a tutorial on support vector machines for pattern recognition, Data Mining \& Knowledge Discovery 2 (2) (1998) 121-167.

[5] J. Yu, D. Tao, R. Hong, X. Gao, Recent developments on deep big vision, Neurocomputing.

[6] F. Melgani, L. Bruzzone, Classification of hyperspectral remote sensing images with support vector machines, Geoscience \& Remote Sensing IEEE Transactions on 42 (8) (2004) 1778 - 1790.

[7] G. Camps-Valls, L. Gomez-Chova, J. Calpe-Maravilla, J. D. Martin-Guerrero, E. Soria-Olivas, L. AlonsoChorda, J. Moreno, Robust support vector method for hyperspectral data classification and knowledge discovery, IEEE Transactions on Geoscience \& Remote Sensing 42 (7) (2004) 1530-1542.

[8] G. M. Foody, A. Mathur, A relative evaluation of multiclass image classification by support vector machines, Geoscience \& Remote Sensing IEEE Transactions on 42 (6) (2004) 1335-1343.

[9] J. A. Gualtieri, R. F. Cromp, Support vector machines for hyperspectral remote sensing classification, Proceedings of SPIE - The International Society for Optical Engineering 3584 (25) (1999) 1-28.

[10] J. Yu, Y. Rui, Y. Y. Tang, D. Tao, High-order distance-based multiview stochastic learning in image classification., IEEE Transactions on Cybernetics 44 (12) (2014) 2431-2442.

[11] E. Zhang, X. Zhang, L. Jiao, H. Liu, S. Wang, B. Hou, Weighted multifeature hyperspectral image classification via kernel joint sparse representation, Neurocomputing 178 (2015) 71-86.

[12] G. R. G. Lanckriet, N. Cristianini, P. Bartlett, L. El Ghaoui, M. I. Jordan, Learning the kernel matrix with semi-definite programming, in: Proceedings of the Nineteenth International Conference on Machine Learning, 2002, pp. 27-72.

[13] R. P. W. D. Wan-Jui Lee, Sergey Verzakov, Kernel combination versus classifier combination, Springer Berlin Heidelberg (2007) 22-31.

[14] S. C. Y. G. Alain Rakotomamonjy, Francis R. Bach, Simplemkl, Journal of Machine Learning Research 9 (3) (2008) 2491-2521. 
[15] P. A.-D. J. Jun Li, Reddy Marpu, Generalized composite kernel framework for hyperspectral image classification, Geoscience and Remote Sensing, IEEE Transactions on 51 (9) (2013) 4816 - 4829.

[16] Z. H. J. Li, Multiple data-dependent kernel for classification of hyperspectral images, Expert Systems with Applications 51 (18) (2015) 11181135.

[17] D. Y.-Y. Z. Yanfeng Gu, Chen Wang, Representative multiple kernel learning for classification in hyperspectral imagery, Geoscience and Remote Sensing, IEEE Transactions on 50 (7) (2012) 2852 - 2865.

[18] Y. Gu, Q. Wang, H. Wang, D. You, Y. Zhang, Multiple kernel learning via low-rank nonnegative matrix factorization for classification of hyperspectral imagery, IEEE Journal of Selected Topics in Applied Earth Observations \& Remote Sensing 8 (6) (2015) 2739-2751.

[19] Y. Gu, C. Wang, D. You, Y. Zhang, S. Wang, Y. Zhang, Representative multiple kernel learning for classification in hyperspectral imagery, IEEE Transactions on Geoscience \& Remote Sensing 50 (7) (2012) 2852-2865.

[20] Y. Gu, H. Liu, Sample-screening mkl method via boosting strategy for hyperspectral image classification, Neurocomputing 173 (2016) 1630-1639.

[21] X. Xie, B. Li, X. Chai, A framework of quasiconformal mapping-based kernel machine with its application to hyperspectral remote sensing, Measurement 80 (2016) 270-280.

[22] C. S.-B. S. Sören Sonnenburg, Gunnar Rätsch, Large scale multiple kernel learning, Journal of Machine Learning Research 7 (2006) (2006) 2006.

[23] S. Amari, S. Wu, Improving support vector machine classifiers by modifying kernal functions, Neural Networks the Official Journal of the International Neural Network Society 12 (6) (1999) 783-789.

[24] H. Xiong, M. N. S. Swamy, M. O. Ahmad, Optimizing the kernel in the empirical feature space, IEEE Transactions on Neural Networks 16 (2) (2005) 460-474.

[25] C. I. Chang, Spectral information divergence for hyperspectral image analysis, in: Geoscience and Remote Sensing Symposium, 1999. IGARSS '99 Proceedings. IEEE 1999 International, 1999, pp. 509511 vol.1.

[26] F. V. D. Meer, Spectral curve shape matching with a continuum removed ccsm algorithm, International Journal of Remote Sensing 21 (16) (2000) 3179-3185.

[27] R. N. Clark, A. J. Gallagher, G. A. Swayze, Material absorption band depth mapping of imaging spectrometer data using a complete band shape least-squares fit with library reference spectra, in: Proceedings of the 2nd Airborne Visible Infrared Imaging Spectrometer (AVIRIS) Workshop, Jet Propulsion Laboratory, Publication, 1990. 
[28] G. Wu, E. Y. Chang, N. Panda, Formulating context-dependent similarity functions., in: Acm International Conference on Multimedia, 2005, pp. 725-734.

[29] S. C. H. Hoi, W. Liu, M. R. Lyu, W. Y. Ma, Learning distance metrics with contextual constraints for image retrieval, in: 2013 IEEE Conference on Computer Vision and Pattern Recognition, 2006, pp. 2072-2078.

[30] J. T. Kwok, I. W. Tsang, Learning with idealized kernels., Proc Icml 1 (2003) 400-407.

[31] I. W. Tsang, P. M. Cheung, J. T. Kwok, Kernel relevant component analysis for distance metric learning, in: In IEEE International Joint Conference on Neural Networks (IJCNN, 2005, pp. 954-959.

[32] Z. Zhang, Learning metrics via discriminant kernels and multidimensional scaling: Toward expected euclidean representation., Icml 2 (2003) 872-879.

[33] M. S. Baghshah, S. B. Shouraki, Kernel-based metric learning for semi-supervised clustering, Neurocomputing 73 (7-9) (2010) 1352-1361.

[34] M. S. Baghshah, S. B. Shouraki, Learning low-rank kernel matrices for constrained clustering, Neurocomputing 74 (1213) (2011) 2201-2211.

[35] E. P. Xing, A. Y. Ng, M. I. Jordan, S. Russell, Distance metric learning, with application to clustering with side-information, Advances in Neural Information Processing Systems (2002) 505-512.

[36] J. V. Davis, B. Kulis, P. Jain, S. Sra, I. S. Dhillon, Information-theoretic metric learning., in: in NIPS 2006 Workshop on Learning to Compare Examples, 2007, pp. 209-216.

[37] B. Alipanahi, M. Biggs, A. Ghodsi, Distance metric learning vs. fisher discriminant analysis, in: Proceedings of the 23rd national conference on Artificial intelligence - Volume 2, 2008.

[38] J. Goldberger, S. T. Roweis, G. E. Hinton, R. Salakhutdinov, Neighbourhood components analysis, Advances in Neural Information Processing Systems 83 (6) (2004) 513-520.

[39] A. Globerson, S. T. Roweis, Metric learning by collapsing classes., Nips 18 (2005) 451-458.

[40] L. W. J. Goldberger, Classification of hyperspectral remote-sensing images using discriminative linear projections, International Journal of Remote Sensing volume 30 (21) (2009) 5605-5617(13).

[41] K. Q. Weinberger, L. K. Saul, Distance metric learning for large margin nearest neighbor classification., Journal of Machine Learning Research 10 (1) (2009) 207-244.

[42] J. Yu, D. Tao, J. Li, J. Cheng, Semantic preserving distance metric learning and applications, Information Sciences 281 (2014) 674-686. 
[43] C. Hong, J. Yu, D. Tao, M. Wang, Image-based three-dimensional human pose recovery by multiview locality-sensitive sparse retrieval, IEEE Transactions on Industrial Electronics 62 (6) (2015) 3742-3751. doi:10.1109/TIE.2014.2378735.

[44] Z. He, L. Liu, R. Deng, Y. Shen, Low-rank group inspired dictionary learning for hyperspectral image classification, Signal Processing 120 (2016) 209-221.

[45] J. Gomez, J. Blasco, E. Molto, G. Camps-Valls, Hyperspectral detection of citrus damage with mahalanobis kernel classifier, Electronics Letters 43 (20) (2007) 1082-1084.

[46] B. D. Bue, An evaluation of low-rank mahalanobis metric learning techniques for hyperspectral image classification, Selected Topics in Applied Earth Observations \& Remote Sensing IEEE Journal of 7 (4) (2014) 1079-1088.

[47] T. M. Cover, Geometrical and statistical properties of systems of linear inequalities with applications in pattern recognition, IEEE Transactions on Electronic Computers ec-14 (3) (1965) 326-334.

[48] J. Muñoz-Marí, G. Camps-Valls, L. Gómez-Chova, J. Calpe-Maravilla, Combination of one-class remote sensing image classifiers, in: Geoscience and Remote Sensing Symposium, 2007. IGARSS 2007. IEEE International, IEEE, 2007, pp. 1509-1512.

[49] S. Xiang, F. Nie, C. Zhang, Learning a mahalanobis distance metric for data clustering and classification, Pattern Recognition 41 (12) (2008) 3600-3612.

[50] Y. F. Guo, S. J. Li, J. Y. Yang, T. T. Shu, L. D. Wu, A generalized foleysammon transform based on generalized fisher discriminant criterion and its application to face recognition, Pattern Recognition Letters 24 (13) (2003) 147158.

[51] G. H. Golub, C. F. Van Loan, Matrix computations, Mathematical Gazette 47 (5 Series II) (1983) 392-396.

[52] M. Zhao, H. Zhang, Z. Zhang, Learning from local and global discriminative information for semisupervised dimensionality reduction, Information Sciences 324 (2013) 286-309.

[53] C. W.-Y. Z. Zhenlin Liu, Yanfeng Gu, A subspace weighting kernel method combining clustering-based grouping for feature extraction in hyperspectral imagery classification, in: Industrial Electronics and Applications (ICIEA), 2011 6th IEEE Conference on, 2011, pp. 2544 - 2547.

[54] J. Cohen, A coefficient of agreement for nominal scales, Educational \& Psychological Measurement 20 (1) (1960) 37-46.

[55] G. Camps-Valls, Kernel-based methods for hyperspectral image classification, Geoscience and Remote Sensing, IEEE Transactions on 43 (6) (2005) 1351 - 1362. 